\title{
Radiological Analysis of COVID-19 Using Computational Intelligence: A Broad Gauge Study
}

\author{
S. Vineth Ligi $\mathbb{D},{ }^{1}$ Soumya Snigdha Kundu $\left(\mathbb{D},{ }^{2}\right.$ R. Kumar $\left(\mathbb{D},{ }^{1}\right.$ R. Narayanamoorthi $\left(\mathbb{D},{ }^{3}\right.$ \\ Khin Wee Lai $\mathbb{D}^{4}{ }^{4}$ and Samiappan Dhanalakshmi ${ }^{1}$ \\ ${ }^{1}$ Department of Electronics and Communication Engineering, College of Engineering and Technology, \\ Faculty of Engineering and Technology, SRM Institute of Science and Technology, SRM Nagar, Kattankulathur, Chengalpattu, \\ Chennai, TN, India \\ ${ }^{2}$ Department of Computer Science Engineering, College of Engineering and Technology, Faculty of Engineering and Technology, \\ SRM Institute of Science and Technology, SRM Nagar, Kattankulathur, Chengalpattu, Chennai, TN, India \\ ${ }^{3}$ Department of Electrical and Electronics Engineering, College of Engineering and Technology, \\ Faculty of Engineering and Technology, SRM Institute of Science and Technology, SRM Nagar, Kattankulathur, Chengalpattu, \\ Chennai, TN, India \\ ${ }^{4}$ Department of Biomedical Engineering, Faculty of Engineering, Universiti Malaya, Kuala Lumpur, Malaysia
}

Correspondence should be addressed to Khin Wee Lai; lai.khinwee@um.edu.my and Samiappan Dhanalakshmi; dhanalas@ srmist.edu.in

Received 13 August 2021; Revised 13 December 2021; Accepted 7 January 2022; Published 23 February 2022

Academic Editor: Cosimo Ieracitano

Copyright $\odot 2022$ S. Vineth Ligi et al. This is an open access article distributed under the Creative Commons Attribution License, which permits unrestricted use, distribution, and reproduction in any medium, provided the original work is properly cited.

Pulmonary medical image analysis using image processing and deep learning approaches has made remarkable achievements in the diagnosis, prognosis, and severity check of lung diseases. The epidemic of COVID-19 brought out by the novel coronavirus has triggered a critical need for artificial intelligence assistance in diagnosing and controlling the disease to reduce its effects on people and global economies. This study aimed at identifying the various COVID-19 medical imaging analysis models proposed by different researchers and featured their merits and demerits. It gives a detailed discussion on the existing COVID-19 detection methodologies (diagnosis, prognosis, and severity/risk detection) and the challenges encountered for the same. It also highlights the various preprocessing and post-processing methods involved to enhance the detection mechanism. This work also tries to bring out the different unexplored research areas that are available for medical image analysis and how the vast research done for COVID-19 can advance the field. Despite deep learning methods presenting high levels of efficiency, some limitations have been briefly described in the study. Hence, this review can help understand the utilization and pros and cons of deep learning in analyzing medical images.

\section{Introduction}

The pandemic brought forth by the coronavirus disease 2019 (COVID-19) not only sustains a devastating response on the well-being and health of the worldwide population but also demands a high rate of monitoring so that it does not extend on its destructive path. A vital aspect of the battle against COVID-19 is the efficient examination of the patients, which can help the infected receive quick treatment and immediate care. As of now, the customary screening process to identify
COVID-19 is the reverse transcriptase-polymerase chain reaction (RT-PCR) test method. This test identifies the presence of SARS-CoV-2 ribonucleic acid (RNA) in respiratory specimen samples (obtained via a range of procedures such as the nasopharyngeal or oropharyngeal swabs) [1]. The RT-PCR test method, despite being effective, has a few shortcomings. It is time-consuming, complicated, and involves a lot of manual labor. All these concerns make it difficult to comb through the highly populated regions where millions have to be tested in a rapid norm. It is also 
seen that the test's sensitivity aspect is highly variable [2, 3]. Radiographic examination was opted as recourse to surmount the difficulties in RT-PCR testing. Chest radiographs (computed tomography (CT) and chest X-ray (CXR) imaging) are imaged and examined by radiologists (as depicted in Figure 1(a)) to find visible references in tandem to the infection.

Preliminary studies discovered that patients affected by COVID-19 infection have abnormalities in chest radiographic images, with a few proposing that radiographic evaluation could be implemented as the principal method for COVID-19 screening in highly populated epidemic areas $[4,5]$. Among radiographs, the CXRs are preferred over CTs [6] as they support rapid triaging in parallel to viral testing while being readily accessible and available. It is also easy to target multiple regions due to its portability. The more outstanding image quality of CT has to be sacrificed to avail these merits. Although the radiographs generated can significantly improve the process, it requires some form of automation. Doctors can hasten the diagnostic procedure, but it still involves scads of manual labor from skilled radiologists, which is not a feasible solution to tackle the large number of COVID-19 patients. To overcome this constraint, there have been constant research attempts utilizing deep learning (DL) methods to find the abnormalities (as seen in Figure 1(b)) in radiographs [7].

Convolutional neural network $(\mathrm{CNN})$ is the primary choice of neural network framework for any DL practitioner working with medical images [8] and radiographs [9] along with vision-based tasks in general such as classification [10], object detection [11], and segmentation [12]. DenseNet, a type of CNN that forms the base to many of the architecture used to recognize COVID-19 from CTs and CXRs, is shown in Figure 2. CNNs stem from the mathematical operation of convolutions (as shown in Figure 3), which expresses the shape modification of functions. The term convolution encompasses the process and its result function. The ability of CNNs to analyze and capture spatial information helps to perform better than most other algorithms present. CNNs generally comprise convolutional layers, pooling layers, and various filters. The architecture usage depends on the demand and size of data available with which it is training. Dropout [13] and transfer learning [14] are commonly used techniques to improve the model's performance. Normalization approaches such as batch normalization [15] and group normalization [16] help improve the model's performance, provide the ability to users in building larger models, and tackle the vanishing gradient problem.

Machine learning (ML) algorithms are generally chosen over DL algorithms to compensate the computational requirements, but in the medical field, time and computational requirements are always traded off for higher standards of precision and hence used in preprocessing [19], feature selection [20], classification [21], and regression [22]. As the COIVD-19 demanded faster results, machine learning avenues have been explored (as shown in Table 1) to account for the global situation constraints. The outline of the application of the COVID-19 detection system in the real world is pictorially represented in Figure 4. In [18], an extensive study comparing K-nearest neighbor (KNN) and support vector machine (SVM) to CNNs was made. The experimentation presented that the DL classifiers trump the machine learning classifiers. Additionally, the DL-based classification methods generate results nearly 5 times faster than the machine learning classifiers. Hence, they are applied in various fields [23-26]. On experimentation with MobileNetV2 [27], the run time and computational power requirements were further reduced. Table 2 lists the history of the networks used.

A simple inference that can be made throughout all the literature is that the DL models (especially CNNs) surmount the ML models due to their capability of capturing spatial information. The spatial correlations are completely absent in general ML methods and hence fail to capture important correlations or key points that are absent while considering the image in a linear aspect. The DL models are ultimately black box models, and the ML methods are easily interpretable. Still, recent explainable methods such as class activation maps $[48,49]$ help remove that barrier and let the user know how the model is providing an output or how the inference is generally created $[50,51]$.

1.1. Review Outline. The following contributions are made through this review study:

(i) A detailed discussion is done with respect to the COVID-19 prediction approaches of the preceding reviews. The study analyses their merits and demerits and provides key insights as well regarding the same. It covers the essential aspects of COVID19 research that the previous studies have missed.

(ii) A systematic comparison is studied encompassing COVID-19 detection techniques about prognosis, diagnosis, and severity/risk detection.

(iii) An extensive discussion on the challenges with regard to fostering high-quality results in detecting COVID-19. Solutions for these challenges are presented alongside as well.

(iv) An in-depth analysis of the pre- and post-processing methods used on the COVID-19 datasets and architectures is provided.

(v) Discussion on the unexplored areas such as metalearning and self-supervised learning and defining the explorable research avenues regarding the same are presented.

(vi) This work emphasizes how most of the research that takes place for COVID-19 can help propagate research for other diseases and medical image analysis in general.

Moving on to Section 2, the discussion is carried out regarding the premier approaches in COVID-19 detection methodologies on diagnosis, prognosis, and severity/risk detection. The inferences collected from reviewing the papers are also noted. Section 3 discusses the merits and limitations of the past reviews, which have described past attempts on detecting COVID-19 through deep learning 


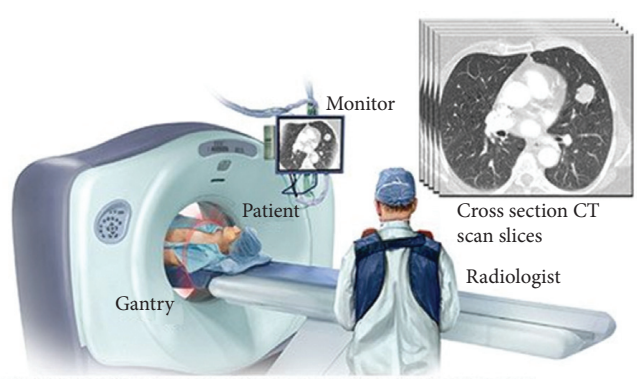

(a)

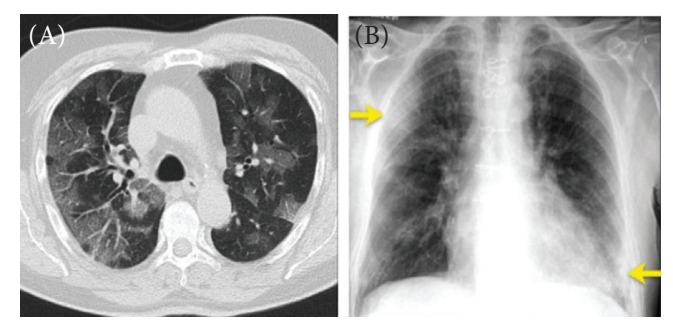

(b)

FIgURE 1: (a) Radiological image acquisition (courtesy: newsnetwork.mayoclinic.org/discussion/mayo-clinic-radio-lung-cancerupdatehousehold-health-hazardsprediabetes). (b) (A) Axial chest CT image (non-enhanced) of a positive RT-PCR-confirmed 70-yearold man showing ground-glass opacities along with dilated segmental and subsegmental vessels prominent on the right side. (b) (B) CXR showing pulmonary hypertension, mitral insufficiency, and atrial fibrillation along with COVID-19 contagion in an 83-year-old man (arrows indicating ground-glass opacity findings in the upper right lobe and consolidation findings in the lower left lobe of the lungs) (arrows) (courtesy: radiologyassistant.nl).

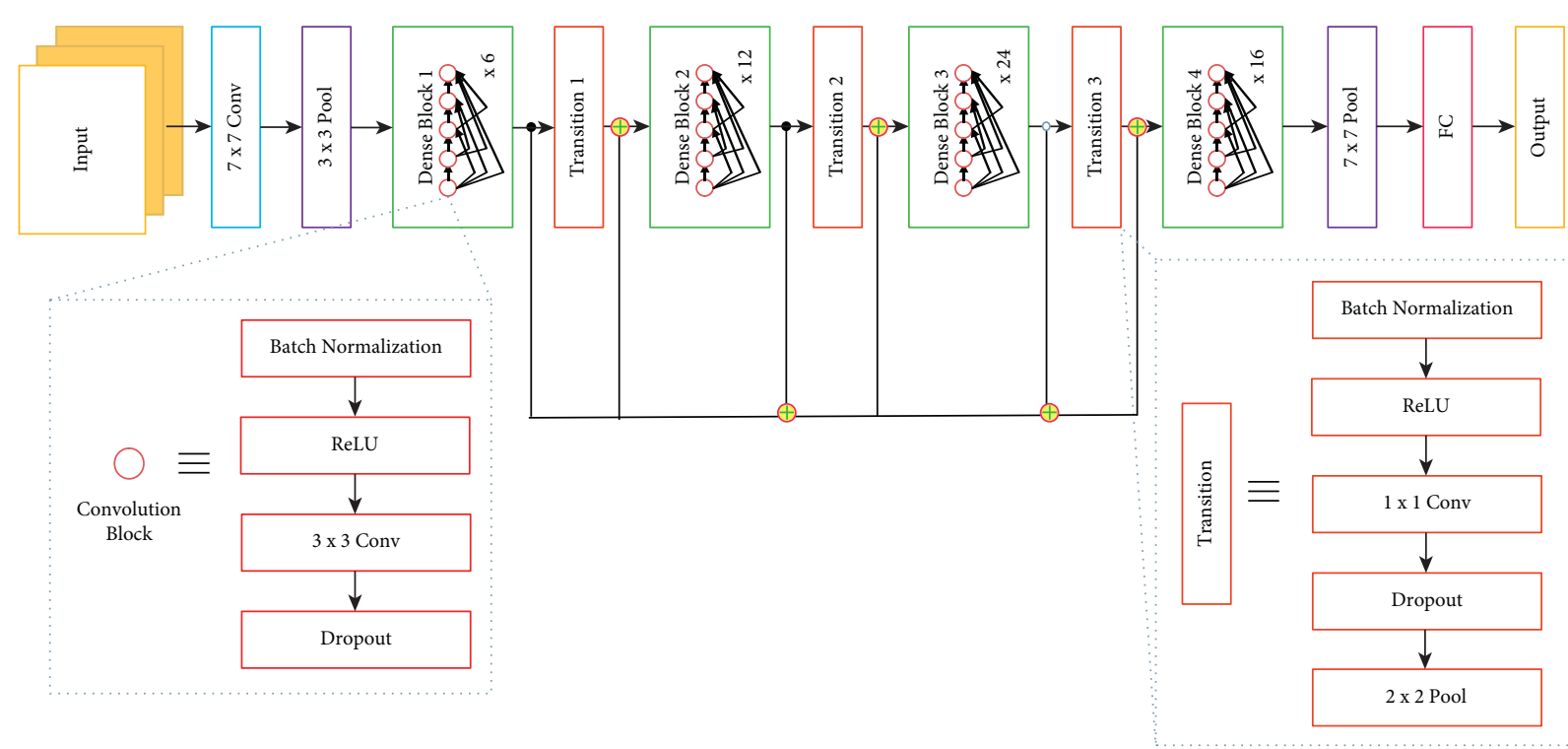

Figure 2: Example of DenseNet architecture.

methods. The section also includes an extension to the solutions provided to the challenges mentioned in Shorten et al. [52] by exploring different paradigms of deep learning. A discussion on the various vision-based pre- and postprocessing techniques used to improve deep learning algorithms is given in Section 4. The review continues by identifying more challenges faced while attempting to detect COVID-19 and a brief discourse on the future directions to utilize the vast research done for COVID-19 towards the domain of medical image analysis in general under Section 5 and the conclusion in Section 6.

\section{Discussion on COVID-19 Medical Image Analysis}

The major COVID-19 medical image analysis tasks are as follows: diagnosis, prognosis, and severity/risk detection. The upsurge of the COVID-19 epidemic has triggered many researchers to contribute their research findings in pulmonary image analysis using DL and other image processing techniques leading to an astonishing breakthrough in COVID-19 diagnosis with stupendous amount of quality works. Discerning COVID-19 from other non-COVID-19 conditions is an important issue to be addressed; hence, the study has been majorly categorized as follows: COVID-19/ non-COVID-19 pneumonia (2-class classification) and COVID-19/non-COVID-19 pneumonia/normal (3-class classification). The study also includes a discussion on classifying COVID-19 against normal condition and other lung diseases, the impact of 2-class and 3-class classifications using the same algorithm on the same dataset, and performing the same classification technique on different image modalities (CT and CXR). The aforementioned workflow has been pictorially depicted in Figure 5 .

2.1. COVID-19 Diagnosis. COVID-19 diagnostic approach based on medical image analysis utilizes the CXR and CT 


\begin{tabular}{|c|c|c|c|c|}
\hline 2 & 4 & 9 & 1 & 4 \\
\hline 2 & 1 & 4 & 4 & 6 \\
\hline 1 & 1 & 2 & 9 & 2 \\
\hline 7 & 3 & 5 & 1 & 3 \\
\hline 2 & 3 & 4 & 8 & 5 \\
\hline
\end{tabular}

\begin{tabular}{|c|c|c|}
\hline 1 & 2 & 3 \\
\hline-4 & 7 & 4 \\
\hline 2 & -5 & 1 \\
\hline
\end{tabular}

Filter/

Kernel

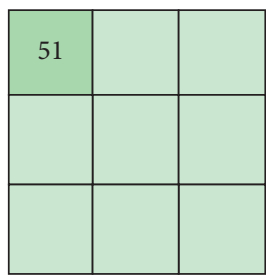

Feature

Image

Figure 3: 2D feature extraction by filters and kernels from images through convolution operations.

TABLE 1: Machine learning techniques tried and true in preceding COVID-19 medical image analysis.

\begin{tabular}{lc}
\hline Algorithm & Summary \\
\hline $\mathrm{RF}^{1}[17]$ & Utilized quantitative features of CT scans \\
SVM [18] & Tested SVM (RBF) ${ }^{1}$ on raw and modified CT images \\
KNN [18] & Tested KNN $(N=21)^{1}$ on raw and modified CT images \\
\hline
\end{tabular}

${ }^{1} \mathrm{RF}$ indicates random forest algorithm. RBF indicates radial basis function. $N$ indicates the number of neighbors considered. Rest all were set to the general settings.

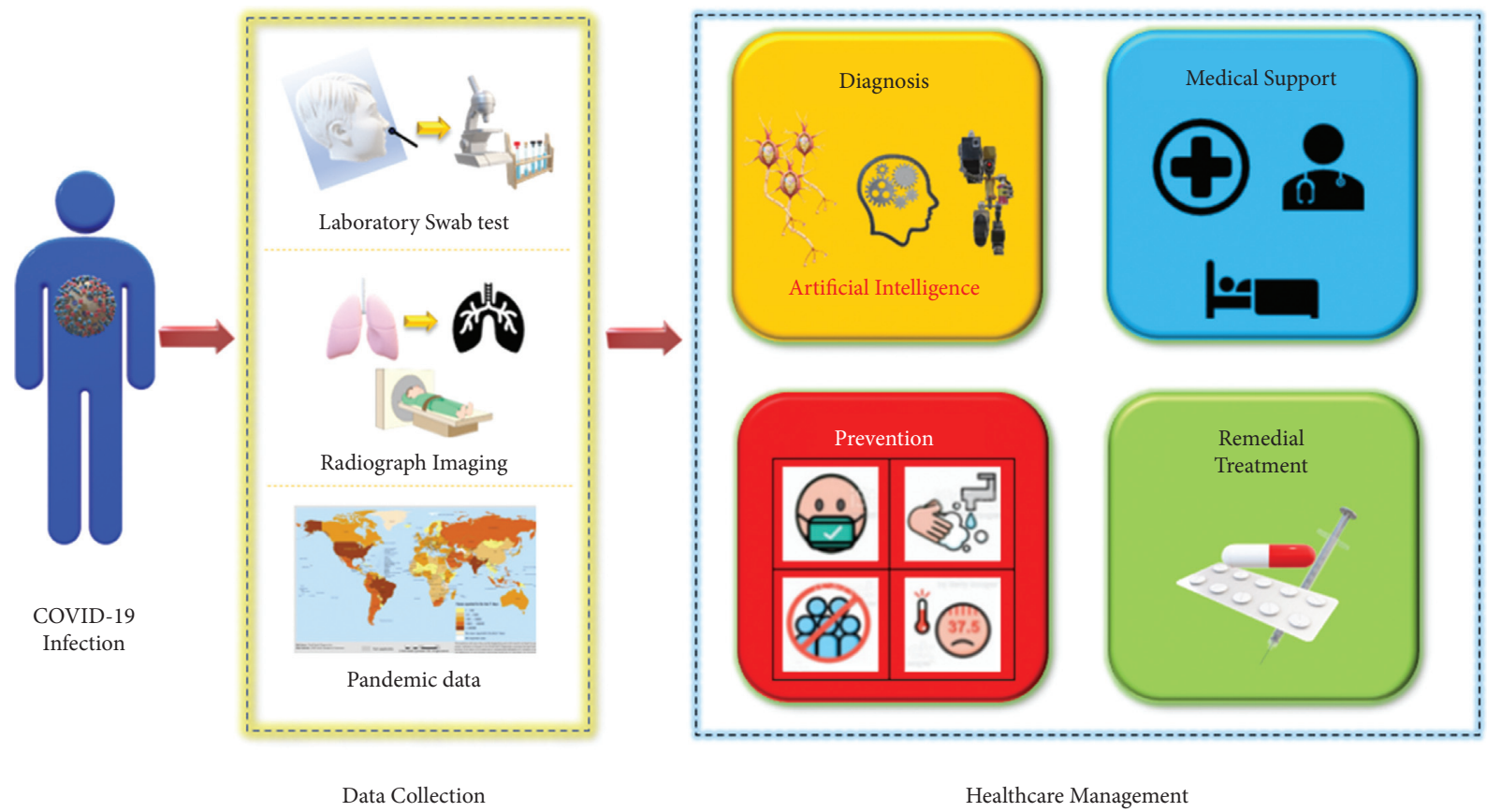

Figure 4: COVID-19 detection system.

images. AI can adequately improve the diagnostic model's efficiency by accurately locating the infections due to the virus in X-ray and CT images, hence facilitating assistance to the radiologists in making clinical decisions for disease diagnosis and triage. [53].

2.1.1. COVID-19/Non-COVID-19 Pneumonia Diagnosis. Harmon et al. [54] proposed an AI-based 3D model using DenseNet-121 to identify COVID-19 from multinational CT data. 2724 scans from 2617 COVID-19 victims were used in this work, among which 1029 scans belonged to 922 RTPCR-confirmed COVID-19 patients. Initially, the lung region was segmented using Anisotropic Hybrid-Net (AHNet) architecture. On testing the model using an independent dataset, it achieved accuracy of $90.8 \%$, area under the curve (AUC) of 0.949 , sensitivity of $84 \%$, specificity of $93 \%$, positive predictive value (PPV) of 0.794 , and negative predictive value (NPV) of 0.984 with sufficient generalization. In 140 non-COVID-19 pneumonia patients, the false- 
TABLE 2: Evolution of CNNs since 1959. The table describes primary points of novelty that motivated new architectures to be produced.

\begin{tabular}{|c|c|c|}
\hline Architecture & Primary focus and novelty & Author and year \\
\hline $\begin{array}{l}\text { Simple and complex cells } \\
\text { [28] }\end{array}$ & $\begin{array}{l}\text { Described cells in the human cortex. } \\
\text { Proposed its use case in pattern recognition. }\end{array}$ & Hubel \& Wiesel (1959) \\
\hline Neocognitron [29] & $\begin{array}{l}\text { Converted the cell idea from [28] into a computational model. } \\
\text { First modern CNN. }\end{array}$ & Fukushima (1980) \\
\hline LeNet-5 [30] & $\begin{array}{l}\text { Composed of two convolution layers with three fully connected layers. Introduced the } \\
\text { MNIST database. } \\
\text { Implemented overlapping pooling and ReLU [32]. }\end{array}$ & Lecun et al. (1998) \\
\hline AlexNet [31] & $\begin{array}{l}\text { Non-saturating neurons are used. } \\
\text { Facilities' effective usage of GPU-driven methods. }\end{array}$ & Krizhevsky et al. (2012) \\
\hline VGG-16 [33] & $\begin{array}{l}\text { Made an exhaustive evaluation on architectures of increasing depth. } \\
\text { Used architectures with tiny }(3 \times 3) \text { convolution filters. } \\
\text { Dimensions of network are increased while keeping the computational budget }\end{array}$ & $\begin{array}{l}\text { Simonyan and } \\
\text { Zisserman (2014) }\end{array}$ \\
\hline Inception [34] & $\begin{array}{l}\text { constant. } \\
\text { Utilized the Hebbian principle and multiscale processing. }\end{array}$ & Szegedy et al. (2015) \\
\hline Modified VGG-16 [35] & $\begin{array}{c}\text { Proposed that if a model is strong enough to fit a large dataset, it can also fit to a small } \\
\text { one. }\end{array}$ & Liu and Deng (2015) \\
\hline ResNet [36] & $\begin{array}{l}\text { Presented a residual learning framework. } \\
\text { Allowed building larger models with deeper layers through skip connections. Paved } \\
\text { the way for more variants }[37,38] \text {. }\end{array}$ & He et al. (2015) \\
\hline Xception [39] & $\begin{array}{c}\text { Presented a depth-wise separable convolution as an inception module with a } \\
\text { maximally large number of towers. }\end{array}$ & Chollet (2016) \\
\hline MobileNets [40] & $\begin{array}{l}\text { Made for mobile and embedded vision applications. } \\
\text { Streamlined architecture using depth-wise separable convolutions. }\end{array}$ & Howard et al. (2017) \\
\hline ResNeXt [41] & $\begin{array}{l}\text { Presented cardinality (size of the transformation set) as a key factor along with the } \\
\text { dimensions of an architecture. }\end{array}$ & Xie et al. (2017) \\
\hline DenseNet [42] & $\begin{array}{l}\text { Complete intra-layer connections among all singular connections in a feed-forward } \\
\text { fashion. } \\
\text { Strengthens feature propagation and encourages feature reuse. }\end{array}$ & Blei et al. (2017) \\
\hline $\begin{array}{l}\text { Squeeze-and-excitation } \\
\text { block [43] }\end{array}$ & $\begin{array}{c}\text { Adaptively recalibrates channel-wise feature responses by explicitly modelling } \\
\text { interdependencies between channels. }\end{array}$ & Hu et al. (2018) \\
\hline Residual inception [44] & Combined residual and inception module. & Zhang et al. (2018) \\
\hline $\begin{array}{l}\text { NASNet search space } \\
{[45]}\end{array}$ & $\begin{array}{l}\text { Designed a new search space to enable transferability. } \\
\text { Presented a new regularization technique-scheduled drop path }\end{array}$ & Zoph et al. (2018) \\
\hline EfficientNet [46] & $\begin{array}{l}\text { Proposed a novel scaling technique that scales all the dimensions (width/resolution/ } \\
\text { depth) uniformly using a compound coefficient. }\end{array}$ & Tan and Le (2019) \\
\hline $\begin{array}{l}\text { Normalizer-free models } \\
{[47]}\end{array}$ & $\begin{array}{c}\text { Developed an adaptive gradient clipping technique to overcome instability. } \\
\text { Designed a significantly improved class. }\end{array}$ & Brock et al. (2021) \\
\hline
\end{tabular}

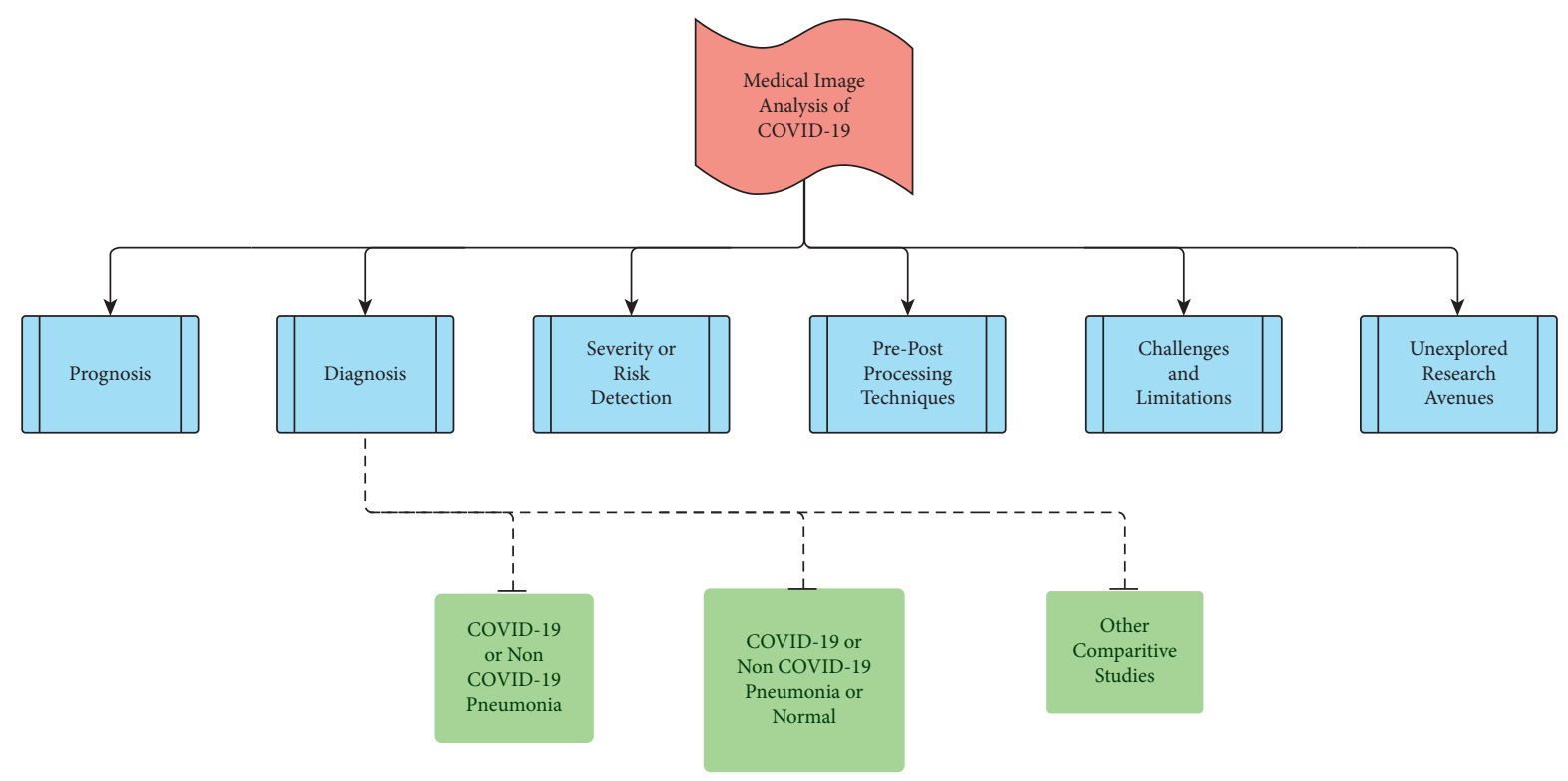

FIgURE 5: Flow of review on different medical imaging analysis tasks. 
positive rate was $10 \%$. This model was able to furnish reasonable performance metrics, thereby enabling it as an unbiased clinical trial tool for assisting the COVID-19 medical image analysis in specific bounded societies during the epidemic outbreak.

A dual-sampling attention network realized by a $3 \mathrm{D}$ CNN using ResNet-34 with an online attention refinement and a dual-sampling strategy was proposed by Ouyang et al. [55] to categorize COVID-19 against community-acquired pneumonia (CAP). The model was evaluated on a multicenter CT dataset of 4982 images consisting of 1593 CAP and 3389 COVID-19 images. Dual-sampling strategy (uniform sampling and size-balanced sampling) was used to mitigate the effect of imbalanced learning and the online attention module to target the infected regions, thereby increasing the model explainability and interpretability by showing visual evidence to reveal the critical regions considered by the model for diagnosis. The ability to generalize the proposed model was evaluated using an autonomous test data, which gave an accuracy of $87.5 \%$, AUC value of 0.944 , along with sensitivity of $86.9 \%$, specificity of $90.1 \%$, and $F 1$ score of $82.0 \%$.

A multiview fusion model using ResNet-50 was developed by Wu et al. [56], which makes use of the axial, sagittal, and coronal views of CT as the three-channel input image to the model. A multicenter dataset consisting of high-resolution CT images of 368 COVID-19-infected patients and 127 patients suffering from other pneumonia (67 viral pneumonia, 47 bacterial pneumonia, 11 mycoplasma pneumonia, and 2 fungal pneumonia) were collected. The multiview model shows better performance than the singleview model with an accuracy of $76 \%$, AUC value of 0.819 , sensitivity of $81.1 \%$, specificity of $61.5 \%$, and overcoming the overfitting issue of the single-view model. This model can mitigate the work burden of radiologists and hence improve the diagnostic efficiency.

Ardakani et al. [57] compared ten convolutional neural networks: ResNet-101, ResNet-50, ResNet-18, VGG-16, VGG-19, MobileNetV2, SqueezeNet, AlexNet, GoogLeNet, and Xception to distinguish COVID-19 against nonCOVID-19 pneumonia. A dataset consisting of 1020 highresolution CT scan slices from 108 COIVD-19 victims and 86 victims with non-COVID-19 pneumonia (viral pneumonia and other atypical pneumonia) were collected. ResNet-101 surpassed the other CNNs due to its high sensitivity of $100 \%$ and AUC value of 0.994 for the given dataset. It also achieved accuracy of $99.51 \%$, specificity of $99.02 \%$, PPV of $99.03 \%$, and NPV of $100 \%$. This model is claimed to remove the substantial cost and can be used as an ancillary method in CT imaging.

An adaptive feature selection-guided deep forest (AFSDF) method was proposed by Sun et al. [58] for COVID19 classification using CT radiographs. The deep forest model was used on four location-specific handcrafted features such as volume, surface area, number of infected lesions, and histogram distribution from the CT images to describe high-level feature representation. The selected features were classified using SVM. A dataset consisting of 2522 de-identified pulmonary CT images from 1027 CAP and 1495 COVID-19-infected patients was used for this study. The proposed AFS-DF variants outperform by achieving $1.38 \%, 1.15 \%$, and $1.11 \%$ enhancement over their obverse methods (logistic regression (LR), SVM, and $\mathrm{RF}$ ) in most of the evaluation metrics. AFS-DF-SVM outperforms the other models with accuracy of $91.79 \%$, AUC value of 0.9635 , sensitivity of $93.05 \%$, and specificity of $89.95 \%$. ASF-DF reduces the repetition of features using the trained forest.

In the study by Narin et al. [59], binary classifications were performed to distinguish COVID-19 against viral and bacterial pneumonia using 341 COVID-19 CXRs and 2800 normal CXRs from the GitHub repository (open source) commonly contributed by Cohen et al. [60] and ChestX-ray8 database [61], respectively. 2772 bacterial pneumonia and 1493 viral pneumonia CXRs were collected from a Kaggle repository called chest X-ray images (pneumonia) [62]. The model performance of five different pre-trained CNN variants (ResNet-152, ResNet-101, ResNet-50, InceptionResNetV2, and InceptionV3) was compared, among which ResNet-50 model showcased the highest classification performance. This model achieved an accuracy, precision, and specificity of $99.5 \%, 98.0 \%$, and $99.5 \%$, respectively, for discriminating COVID-19 against other viral pneumonia, whereas for COVID-19/bacterial pneumonia classification, the accuracy, precision, and specificity values were $99.7 \%$, $98.3 \%$, and $99.8 \%$, respectively. This method was implemented directly in an end-to-end manner eliminating manual intervention for feature extraction, feature selection, or classification tasks.

A deep learning model comprising of three major components, a backbone network, a classification head, and an anomaly detection head, was proposed by Zhang et al. [63] to reduce the false-negative rate as much as possible. The model was built using 1431 CXR pneumonia images of 1008 patients from the ChestX-ray14 dataset [61] and 100 images belonging to 70 COVID-19 patients from the GitHub repository [60]. The experiment was conducted for different values of $T$ parameter that controls the compensation between the true-positive rate and the true-negative rate. As the $T$ value decreases from 0.50 to 0.15 , the sensitivity increases from $72.00 \%$ to $96.00 \%$ and the specificity drops from $97.97 \%$ to $70.65 \%$, but the AUC value remains the same. Based on the performance metrics (sensitivity-96\%, specificity-70.65\%, and AUC-95.18), the model performs well for $T=0.15$ with a reduced false-negative rate of nearly $4 \%$. Despite its good performance, it had its limitations, $4 \%$ missing COVID-19 cases and a false-positive rate of almost $30 \%$.

Abraham and Nair [64] used a combination of multiCNN models (MobileNetV2, SqueezeNet, Xception, DarkNet-53, and ShuffleNet) with correlation-based feature selection (CFS) followed by the BayesNet classifier. The experiment was performed using two datasets: Dataset I: 453 COVID-19 and 107 non-COVID-19 images of either bacterial/viral pneumonia [55] and 390 CXRs of viral and bacterial pneumonia $[60,61,65,66]$ and Dataset II: 71 COVID-19 CXRs and 7 non-COVID-19 CXRs [66]. Only the BayesNet classifier achieved an accuracy of $>90 \%$. The 
proposed model gave an accuracy of $91.16 \%$ and $91.44 \%$ for Dataset I and Dataset II, respectively. The multi-CNNs (with 3 or more pre-trained CNNs) comparatively showed better results than the single pre-trained CNNs.

Autee et al. [67] proposed the StackNet-DenVIS to reduce the false-negative rate in classification using a stacked generalization ensemble of four different CNNs. A total of 9953 CXRs consisting of 9085 non-COVID-19 and 868 COVID-19 cases from multiple sources were gathered $[60,65,68,69]$. The data imbalance problem was handled by generating synthetic images using deep convolutional adversarial generative networks and SMOTE + Tomex links. With an accuracy of $95.07 \%$, this model achieved a low falsenegative rate at low cost in comparison with the RT-PCR test.

2.1.2. COVID-19/Non-COVID-19 Pneumonia/Normal or Non-Pneumonia Diagnosis. A 3D deep learning framework, COVNet, realized using ResNet-50 was implemented by $\mathrm{Li}$ et al. [70] to distinguish COVID-19 against CAP and nonpneumonia cases. The network was able to extract the $2 \mathrm{D}$ local features and the 3D global representative features for better classification. A CT image dataset consisting of 1292 COVID-19 CTs, 1325 CTs with non-COVID-19 pneumonia, and 1735 CTs with CAP infections, totally contributing to 4352 scans, was collected for this study. An AUC value of 0.96 , sensitivity of $90 \%$, and specificity of $96 \%$ were obtained with $95 \%$ confidence interval for an independent test dataset. Due to the shortage of laboratory-confirmed COVID-19 data, the work was unable to present the results for distinguishing COVID-19 from other lung diseases.

Wang et al. [71] have proposed a prior-attention residual model, PA-66-M, using two subnetworks based on 3D ResNets for pneumonia detection and classification of pneumonia type. The two subnetworks were integrated by a late-fusion strategy using a fully connected layer with learning capacity. Lung segmentation was performed using U-Net. The dataset consisting of 936 chest CTs of normal cases, 2406 CT images with interstitial lung disease (only viral pneumonia), and 1315 COVID-19-infected CT images was collected from multiple cooperative hospitals. The proposed model was capable of accurately focusing the lesions with an accuracy, sensitivity, and specificity of $93.3 \%$, $87.6 \%$, and $95.5 \%$, respectively. By applying a constant weighting factor, the prior-attention residual model was able to converge faster than the self-attention strategy. Some of the normal scans were misclassified to pneumonia class by the proposed model, and it also failed to unveil some of the scans with COVID-19 lesions.

Hasan et al. [72] used handcrafted texture features based on Q-deformed entropy along with deep features from $\mathrm{CNN}$. The extracted features were refined by analysis of variance (ANOVA) and then classified to distinguish COVID-19 from other pneumonia types and normal cases. The LSTM neural network classifier outperformed SVM, $\mathrm{KNN}$, and LR with an accuracy of $99.68 \%$. The performance of the combined features was better when compared to using only handcrafted or deep features.
Three-dimensional classification models using two CNNs namely the ResNet-23 and ResNet-18 were used by Butt et al. [73] to classify COVID-19/influenza A viral pneumonia (IAVP)/normal CT image patches. A location attention mechanism was incorporated to identify the corresponding location of the identified patch in the pulmonary CT image. This model was smart enough to accurately distinguish COVID-19, when compared to using a model without location attention mechanism. Hence, an overall accuracy of $86.7 \%$ was observed with sensitivity of $98.2 \%$, specificity of $92.2 \%$, and AUC value of 0.996 . This work used 618 transverse-section CT samples in which 219 samples were obtained from nearly 110 COVID-19-infected patients, 224 scans from 224 IAVP patients, and 175 from healthy people.

Detailed relation extraction neural network (DRENet) is a pre-trained ResNet-50 with feature pyramid network proposed by Song et al. [74] to derive the top-K-level features and extract the image-level predictions for COVID-19 diagnosis at the patient level. For model development and evaluation, the dataset was collected from different hospitals comprising 777 CT images from 88 COVID- 19 victims, 505 CT slices from 100 bacterial pneumonia patients, and $708 \mathrm{CT}$ slices from 86 healthy people. The regions detected by the proposed model contained the most important feature of COVID-19 infection, ground-glass opacity (GGO). DRENet exhibited an efficient performance with an accuracy of $93 \%$ and F1 score of 0.93 .

A social mimic optimization method was proposed by Toğaçar et al. [75] to select the potential deep features from the combined feature set of MobileNetV2 and SqueezeNet, to categorize COVID-19 from pneumonia and normal conditions. It provides efficient features by stacking the original images with the reconstructed fuzzy color images, which had better quality and reduced noise. It used 76 COVID-19 images from [60] and 295 COVID-19, 98 pneumonia, and 65 normal images from [69]. On classification using SVM, all performance metrics, F score, sensitivity, specificity, precision, and accuracy, were $100 \%$ for detecting COVID-19 cases, exhibiting an overall accuracy of 99.27\%. The average values of F score, sensitivity, specificity, and precision for all the three classes are $0.9858,98.33 \%$, $99.69 \%$, and $98.89 \%$, respectively. The model was aimed to produce swift and more authentic results as MobileNetV2 and SqueezeNet used fewer parameters compared with the other networks.

Wang et al. [68] created an open-source network, COVID-Net, and public dataset, COVIDx, consisting of 13,975 CXR images belonging to 13,870 patients obtained by combining data from five different public data repositories [60, 76-79]. COVID-Net architecture used a lightweight residual design pattern called projection-expansion-projection-extension (PEPX) pre-trained on ImageNet dataset.

Compared with VGG-19 and ResNet-50 architectures, COVID-Net has lower complexity in terms of architecture and computations. It showed an accuracy of $93.3 \%$ with sensitivity of $91.0 \%$. Qualitative analysis of the network implies that it does not depend on inappropriate information for decision-making. 
Nishio et al. [80] have evaluated the performance of conventional neural network architectures with different data augmentation techniques (conventional method, mix up, and random image cropping and patching (RICAP)) to identify COVID-19 pneumonia from pulmonary X-rays. 215 COVID-19-infected, 533 non-COVID-19 pneumonia-infected, and 500 healthy CXR images $[60,80]$ were used for this work. VGG-16 with the combination of conventional with mix-up data augmentation was found to give better results with an accuracy of $83.7 \%$ and a sensitivity value of 90.9\% compared with ResNet-50, DenseNet-121, MobileNet, and EfficientNet.

The MH-Net proposed by Canayaz et al. [81] makes use of two meta-heuristic algorithms namely the binary gray wolf optimization (BGWO) and binary particle swarm optimization (BPSO) to select the potential features extracted from VGG-19, ResNet, GoogLeNet, and AlexNet. Finally, an SVM classifier was used. 364 CXR images each of COVID-19, pneumonia, and normal cases $([60,65,69])$ enhanced by the image contrast enhancement algorithm were used for this work. VGG-19 model with BPSO feature optimization (488 features) on the enhanced data outperforms the other models with an overall accuracy of $99.38 \%$, sensitivity of $99.39 \%$, and specificity of $99.69 \%$. The unbalanced class problem is overcome using equal number of CXRs in each class, and also, it uses fewer parameters compared with other models.

The COVID-19 Inception-ResNet model (CoVIRNet) that uses different inception residual blocks for diagnosing COVID-19 infection from the CXR images was proposed by Almalki et al. [82]. Multiscale feature maps obtained from different depths, which are then concatenated by average pooling, are used to improve the efficiency of the proposed method. The problem of overfitting encountered by small datasets has been overcome using different regularization techniques in the deep learning blocks. The author proposed two approaches: (i) CoVIRNet-Inception-ResNet blocks consisting of a single inception module with extra branches of convolution layer using reduction factorization; (ii) CoVIRNet with RF-multiscale, multilayer features extracted from the proposed Inception-ResNet blocks are classified using a random forest classifier. For this, a multicenter dataset of size of 1251 was used, among which 284 COVID19 infection images were collected from [79, 83]. 310 normal CXRs, 330 bacterial pneumonia, and 327 viral pneumoniainfected images were collected from [62]. On comparing the performance of CoVIRNet with fine-tuned versions of Xception, ResNet-101, MobileNetV2, and DenseNet-201, the second approach CoVIRNet with RF showed better performance with accuracy of $97.29 \%$, precision of $97.74 \%$, recall of $97.02 \%$, and $F$ score of 0.9732 .

Subsection 2.1 gives a brief review on the different COVID-19 diagnostic methods by performing two-class or three-class classifications against other pneumonia/normal cases proposed by various researchers in both CXR and CT imaging modalities. Tables 3 and 4 summarize the studies including the network, dataset, and performance metrics used for the evaluation of COVID-19/non-COVID-19 pneumonia diagnosis and COVID-19/non-COVID-19 pneumonia/normal or non-pneumonia diagnosis, respectively. The performance metrics used for evaluation and their corresponding formulae are tabulated in Table 5.

2.1.3. Other Comparative Studies. Apart from the techniques mentioned in the previous sections, there are also other comparative studies done by some researchers, which explore the performance of a COVID-19 diagnostic algorithm for different image modalities (CT and CXR) or on datasets with binary (COVID-19/non-COVID-19) or multiclass classifications (COVID-19/non-COVID-19/normal/ other lung diseases).

(1) Comparison of Binary and Multiclass Classification. $\mathrm{Hu}$ et al. [84] performed an automated diagnosis of COIVD-19 based on ShuffleNetV2 on pulmonary CT images. Two classifications are performed on the data collected from multiple sources. 16 different data augmentation operations were performed on the 1042 chest CT images (comprising of 521 COVID-19, 397 healthy, 76 bacterial pneumonia, and 48 SARS) to increase the dataset size for better training of the model. Binary classification of COVID-19 from the healthy cases obtained an accuracy of $91.21 \%$ along with sensitivity of $90.52 \%$, specificity of $91.58 \%$, and AUC value of 0.9689 . In the case of multiclass classification (COVID-19/bacterial pneumonia/SARS), the accuracy dropped to $85.40 \%$ for the same algorithm. The sensitivity, specificity, and AUC values were $85.71 \%, 84.88 \%$, and 0.9222 , respectively.

Chowdhury et al. [69] had compared the performance of different pre-trained CNNs for COVID-19 detection with and without data augmentation using the data collected from multiple public datasets $([60,83,85])$. Among the various networks analyzed, DenseNet-201 showed comparably better classification results for both COVID-19/normal and COVID-19/normal/pneumonia discrimination with image augmentation. The binary classification shows better performance with an accuracy of $99.7 \%$ compared with the multiclass problem with an accuracy of $97.94 \%$. The performance difference was insignificant, and the overall performance of three-class problem was less in comparison with the binary classification problem.

COVID-DenseNet proposed by Sarker et al. [86] is a deep learning architecture realized using DenseNet-121 with transfer learning from CheXNet for the detection of COVID-19 from COVIDx [71] CXR images. The most significant regions in the image that were responsible for the prediction were highlighted by performing an interpretation analysis using Grad-CAM. The overall accuracy for COVID19/non-COVID-19 classification and COVID-19/pneumonia/normal classification is 0.96 and 0.94 , respectively. This work tried to make the model explainable and interpretable to certain extent using the Grad-CAM representation.

DarkCovidNet architecture based on the DarkNet-19 model was designed by Ozturk et al. [87] to identify COVID19 from X-ray images collected from $[60,61]$ comprising of 127 COVID-19, 500 pneumonia, and 500 normal images. For COVID-19/no findings/pneumonia classification, the model produced a classification accuracy, sensitivity, and 
TABLE 3: A summary of research reviewed on COVID-19/non-COVID-19 pneumonia diagnosis.

\begin{tabular}{|c|c|c|c|c|c|c|c|c|c|}
\hline Work & $\begin{array}{l}\text { Image } \\
\text { modality }\end{array}$ & Dataset size & Method used & $\begin{array}{l}\text { Accuracy } \\
\text { (in \%) }\end{array}$ & $\begin{array}{l}\text { Sensitivity or } \\
\text { recall (in \%) }\end{array}$ & $\begin{array}{l}\text { Specificity } \\
\text { (in \%) }\end{array}$ & $\begin{array}{l}\text { AUC } \\
\text { (in \%) }\end{array}$ & $\begin{array}{l}\text { Precision } \\
\text { (in \%) }\end{array}$ & $\begin{array}{c}\text { F1 } \\
\text { score }\end{array}$ \\
\hline $\begin{array}{l}\text { Harmon } \\
\text { et al. [54] }\end{array}$ & CT & $\begin{array}{l}\text { (i) } 1029 \\
\text { COVID-19 } \\
\text { (ii) } 1695 \text { non- } \\
\text { COVID-19 } \\
\text { Pneumonia }\end{array}$ & $\begin{array}{c}\text { DenseNet-121 and } \\
\text { AH-Net } \\
\text { segmentation }\end{array}$ & 90.8 & 84 & 93 & 94.9 & NA & NA \\
\hline $\begin{array}{l}\text { Ouyang et al. } \\
{[55]}\end{array}$ & CT & $\begin{array}{l}\text { (i) } 3389 \\
\text { COVID-19 } \\
\text { (ii) } 1593 \text { CAP }\end{array}$ & $\begin{array}{l}\text { Dual sampling } \\
\text { Attention network } \\
\text { with ResNet-34 }\end{array}$ & 87.5 & 86.9 & 90.1 & 94.4 & NA & 0.82 \\
\hline $\begin{array}{l}\text { Wu et al. } \\
{[56]}\end{array}$ & CT & $\begin{array}{l}\text { (i) } 331 \\
\text { COVID-19 } \\
\text { (ii) } 114 \text { other } \\
\text { pneumonia }\end{array}$ & $\begin{array}{l}\text { Multiview fusion } \\
\text { model using } \\
\text { ResNet-50 }\end{array}$ & 76 & 81.1 & 61.5 & 81.9 & NA & NA \\
\hline $\begin{array}{l}\text { Ardakani } \\
\text { et al. [57] }\end{array}$ & CT & $\begin{array}{l}\text { (i) } 510 \\
\text { COVID-19 } \\
\text { (ii) } 510 \text { non- } \\
\text { COVID-19 }\end{array}$ & ResNet-101 & 99.51 & 100 & 99.02 & 99.4 & NA & NA \\
\hline $\begin{array}{l}\text { Sun et al. } \\
{[58]}\end{array}$ & CT & $\begin{array}{l}\text { (i) } 1495 \\
\text { COVID-19 } \\
\text { (ii) } 1027 \text { CAP }\end{array}$ & $\begin{array}{l}\text { Adaptive feature } \\
\text { Selection-guided } \\
\text { deep forest-SVM }\end{array}$ & 91.79 & 93.05 & 89.95 & 96.35 & NA & NA \\
\hline $\begin{array}{l}\text { Narin et al. } \\
\text { [59] }\end{array}$ & CXR & $\begin{array}{l}\text { (i) } 341 \\
\text { COVID-19 } \\
\text { (ii) } 1493 \text { viral } \\
\text { pneumonia } \\
\text { (iii) } 341 \\
\text { COVID-19 } \\
\text { (iv) } 2772 \\
\text { bacterial } \\
\text { pneumonia } \\
\end{array}$ & ResNet-50 & 99.7 & 99.4 & 99.8 & $\mathrm{NA}$ & 98.3 & 0.987 \\
\hline $\begin{array}{l}\text { Zhang et al. } \\
{[63]}\end{array}$ & CXR & $\begin{array}{l}\text { (i) } 100 \\
\text { COVID-19 } \\
\text { (ii) } 1431 \\
\text { pneumonia }\end{array}$ & $\begin{array}{c}\text { Residual CNN with } \\
\text { anomaly detection } \\
\text { head }\end{array}$ & NA & 96 & 70.65 & 95.18 & NA & NA \\
\hline $\begin{array}{l}\text { Abraham } \\
\text { and Nair } \\
{[64]}\end{array}$ & CXR & $\begin{array}{l}\text { (i) } 453 \\
\text { COVID-19 } \\
\text { (ii) } 497 \text { non- } \\
\text { COVID-19 } \\
\text { Pneumonia } \\
\text { (i) } 71 \text { COVID- } \\
19 \\
\text { (ii) } 7 \text { non- } \\
\text { COVID-19 } \\
\text { Pneumonia }\end{array}$ & $\begin{array}{c}\text { Combination of } \\
\text { multi-CNN }\end{array}$ & 91.44 & 98.6 & NA & 96.3 & 98.6 & 0.914 \\
\hline $\begin{array}{l}\text { Autee et al. } \\
{[67]}\end{array}$ & CXR & $\begin{array}{l}\text { (i) } 868 \\
\text { COVID-19 } \\
\text { (ii) } 9085 \text { non- } \\
\text { COVID-19 }\end{array}$ & StackNet-DenVIS & 95.07 & 99.40 & 94.61 & 98.40 & NA & NA \\
\hline
\end{tabular}

Bold values represent the best result obtained for each performance metric among all the methodologies compared.

specificity of $87.02 \%, 85.35 \%$, and $92.18 \%$, respectively. In the case of binary classification COVID-19/no findings, the performance metrics increased to accuracy of $98.08 \%$, sensitivity of $95.13 \%$, and specificity of $95.3 \%$. DarkCovidNet was able to diagnose COVID-19 within seconds.

Mahmud et al. [88] proposed the CovXNet architecture, which is a multi-dilation CNN architecture that makes use of transferable multi-receptive feature optimization technique for COVID-19 detection from CXR images. A balanced dataset consisting of 305 images of different resolutions collected from different medical centers was used for each class: COVID-19, viral pneumonia, bacterial pneumonia, and normal. For distinguishing COVID-19 against normal, bacterial pneumonia, and viral pneumonia, the binary classification resulted in a accuracy of $97.4 \%, 94.7 \%$, and $87.3 \%$, respectively. While carrying over the same architecture for 
TABLE 4: A summary of research reviewed on COVID-19/non-COVID-19 pneumonia/normal or non-pneumonia diagnosis.

\begin{tabular}{|c|c|c|c|c|c|c|c|c|c|}
\hline Work & $\begin{array}{c}\text { Image } \\
\text { modality }\end{array}$ & Dataset size & Method used & $\begin{array}{l}\text { Accuracy } \\
\text { (in \%) }\end{array}$ & $\begin{array}{c}\text { Sensitivity or } \\
\text { recall (in \%) }\end{array}$ & $\begin{array}{l}\text { Specificity } \\
\text { (in \%) }\end{array}$ & $\begin{array}{l}\text { AUC } \\
\text { (in \%) }\end{array}$ & $\begin{array}{l}\text { Precision } \\
\text { (in \%) }\end{array}$ & $\begin{array}{c}\text { F1 } \\
\text { score }\end{array}$ \\
\hline $\begin{array}{l}\text { Li et al. } \\
{[70]}\end{array}$ & CT & $\begin{array}{l}\text { (i) } 1292 \\
\text { COVID-19 } \\
\text { (ii) } 16325 \text { non- } \\
\text { COVID-19 } \\
\text { pneumonia } \\
\text { (iii) } 1735 \text { CAP }\end{array}$ & COVNet & NA & 90 & 96 & 96 & NA & NA \\
\hline $\begin{array}{l}\text { Wang } \\
\text { et al. [71] }\end{array}$ & CT & $\begin{array}{l}\text { (i) } 1315 \\
\text { COVID-19 } \\
\text { (ii) } 963 \text { normal } \\
\text { (iii) } 2406 \text { ILD }\end{array}$ & $\begin{array}{c}\text { Prior-attention } \\
\text { Residual model 3D } \\
\text { ResNets }\end{array}$ & 93.3 & 87.6 & 95.5 & NA & NA & NA \\
\hline $\begin{array}{l}\text { Hasan } \\
\text { et al. [72] }\end{array}$ & $\mathrm{CT}$ & $\begin{array}{l}\text { (i) } 118 \text { COVID- } \\
19 \\
\text { (ii) } 96 \\
\text { pneumonia } \\
\text { (iii) } 107 \text { normal }\end{array}$ & $\begin{array}{l}\text { LSTM using Q- } \\
\text { deformed entropy } \\
\text { and deep features }\end{array}$ & 99.68 & NA & NA & NA & $\mathrm{NA}$ & NA \\
\hline $\begin{array}{l}\text { Butt et al. } \\
{[73]}\end{array}$ & CT & $\begin{array}{l}\text { (i) } 219 \text { COVID- } \\
19 \\
\text { (ii) } 224 \text { IAVP } \\
\text { (iii) } 175 \text { normal }\end{array}$ & $\begin{array}{l}\text { 3D ResNets with } \\
\text { location attention } \\
\text { mechanism }\end{array}$ & 86.7 & 98.2 & 92.2 & 99.6 & 81.3 & 0.839 \\
\hline $\begin{array}{l}\text { Song et al. } \\
{[74]}\end{array}$ & CT & $\begin{array}{l}\text { (i) } 777 \text { COVID- } \\
19 \\
\text { (ii) } 505 \text { bacterial } \\
\text { pneumonia } \\
\text { (iii) } 708 \text { normal }\end{array}$ & DRENet & 93 & 93 & NA & NA & 93 & 0.93 \\
\hline $\begin{array}{l}\text { Toğaçar } \\
\text { et al. [75] }\end{array}$ & CXR & $\begin{array}{l}\text { (i) } 371 \text { COVID- } \\
19 \\
\text { (ii) } 98 \\
\text { pneumonia } \\
\text { (iii) } 65 \text { normal }\end{array}$ & $\begin{array}{c}\text { SVM-social } \\
\text { Mimic optimized } \\
\text { deep features }\end{array}$ & 99.27 & 98.33 & 99.69 & NA & 98.89 & 0.9858 \\
\hline $\begin{array}{l}\text { Wang } \\
\text { et al. [68] }\end{array}$ & CXR & $\begin{array}{l}\text { (i) } 358 \text { COVID- } \\
19 \\
\text { (ii) } 5538 \text { non- } \\
\text { COVID-19 } \\
\text { pneumonia } \\
\text { (iii) } 8066 \\
\text { normal }\end{array}$ & COVID-Net & 93.3 & 91 & NA & NA & NA & NA \\
\hline $\begin{array}{l}\text { Nishio } \\
\text { et al. [80] }\end{array}$ & CXR & $\begin{array}{l}\text { (i) } 215 \text { COVID- } \\
19 \\
\text { (ii) } 533 \text { non- } \\
\text { COVID-19 } \\
\text { pneumonia } \\
\text { (iii) } 500 \text { normal }\end{array}$ & $\begin{array}{c}\text { VGG-16 with } \\
\text { conventional and } \\
\text { mix-up } \\
\text { augmentation }\end{array}$ & 83.7 & 90.9 & $\mathrm{NA}$ & NA & NA & NA \\
\hline $\begin{array}{l}\text { Canayaz } \\
{[81]}\end{array}$ & CXR & $\begin{array}{l}\text { (i) } 364 \text { COVID- } \\
19 \\
\text { (ii) } 364 \\
\text { pneumonia } \\
\text { (iii) } 364 \text { normal }\end{array}$ & MH-Net & 99.38 & 99.39 & 99.69 & NA & 99.39 & 0.9938 \\
\hline $\begin{array}{l}\text { Almalki } \\
\text { et al. [82] }\end{array}$ & CXR & $\begin{array}{l}\text { (i) } 284 \text { COVID- } \\
19 \\
\text { (ii) } 327 \text { viral } \\
\text { pneumonia } \\
\text { (iii) } 330 \\
\text { bacterial } \\
\text { pneumonia } \\
\text { (iv) } 504 \text { normal }\end{array}$ & $\begin{array}{l}\text { CoVIRNet feature } \\
\text { extractor with RF }\end{array}$ & 97.29 & 97.02 & $\mathrm{NA}$ & $\mathrm{NA}$ & 97.74 & 0.9732 \\
\hline
\end{tabular}

Bold values represent the best result obtained for each performance metric among all the methodologies compared. 
Table 5: Performance metrics used in COVID-19 detection.

\begin{tabular}{lccccc}
\hline Performance metric & Accuracy & Sensitivity/recall & Specificity & Precision & F1 score \\
\hline Formula & $(\mathrm{TP}+\mathrm{TN}) /(\mathrm{TP}+\mathrm{FP}+\mathrm{TN}+\mathrm{FN})$ & $\mathrm{TP} /(\mathrm{FN}+\mathrm{TP})$ & $\mathrm{TN} /(\mathrm{FP}+\mathrm{TN})$ & $\mathrm{TP} /(\mathrm{FP}+\mathrm{TP})$ & $(2 *(R * P)) /(R+P)$ \\
\hline
\end{tabular}

$\mathrm{TP}-$ true positive, $\mathrm{TN}-$ true negative, $\mathrm{FP}-$ false positive, $\mathrm{FN}-$ false negative, $R$-recall, and $\mathrm{P}-$ precision.

multiclass classifications such as COVID-19/viral pneumonia/bacterial pneumonia and COVID-19/normal/viral pneumonia/bacterial pneumonia, the classification accuracies dropped to $89.6 \%$ and $90.2 \%$, respectively. Based on the obtained results, distinguishing viral pneumonia from COVID-19 is arduous when compared to other diseases. CovXNet is highly scalable with huge receptive capacity.

(2) Comparison of COVID-19 Diagnostic Performance in CT and CXR Images. COVID-MTNet is a deep learning architecture proposed by Alom et al. [89] to perform multiple tasks such as COVID-19 segmentation and detection from CT and CXR images. A dataset of 3875 samples of COVID19 pneumonia and 1341samples for normal cases was collected from $[60,62]$. The infected regions were segmented using the NABLA-N network, and the detection process was performed using the inception recurrent residual neural network (IRRCNN) model with transfer learning. The segmentation network using pixel-level analysis significantly reduced the possibility of false-positive and false-negative detections. The model produced a segmentation accuracy of 94.52\% for CXR images and 99.56\% for CT images in the test data. In the detection model, an accuracy of $98.78 \%$ and $87.26 \%$ was observed in the CT and CXR images, respectively. These results show that the CT imaging modality better discriminates the COVID-19 infection from the normal cases. The detection model can be generalized and made to produce more accurate results by training greater number of samples. Some false-positive detections were observed in the segmentation model for CT images due to the insufficiency of labeled CT data for COVID-19 infection.

Vinod and Prabaharan [90] proposed an artificial intelligence technique for fast COIVD-19 diagnosis using decision tree classifier with deep CNN features. The CXR dataset contains 113 normal images and 306 COVID-19infected X-rays. The CT dataset contains 350 COVID-19 images and 395 non-COVID-19 images. The test score resulted in 0.82 for CT and 0.87 for CXR. The recall score is high in the case of CT images, i.e., 0.93 . The recall score for COVID-19 diagnosis in CXR images is 0.88. The number of false negatives is less for diagnosis in CT image modalities.

In [91] by Perumal et al., Haralick texture features were extracted from the enhanced images. These modified images were then fed into different predefined CNN models such as ResNet-50, VGG-16, and InceptionV3 to find the patterns similar to other pneumonia, so that it can easily detect COVID-19 across other diseases. 14 Haralick features (mean, variance, entropy, etc.) were used for the identification of the relationship between biological features in the data. This method was experimented on data from multiple centers $([60,65,92,93])$. VGG-16 using transfer learning achieves better classification with an accuracy of $93 \%$, precision of $91 \%$, and recall of $90 \%$.
Irfan et al. [94] developed a hybrid multimodel deep neural network (HDNN) for COVID-19 detection from multimodal data. It was designed as a mixture of LSTM and $\mathrm{CNN}$ to predict the risk of disease onset from both CT and CXRs. 1500 images from healthy patients and 3500 images from infected (COVID-19 and pneumonia) patients were collected from various sources $([60,77,78,95,96])$. Initial preprocessing involves the Kalman discrete-time modelbased denoising followed by sampling the $1080 \times 1080$ sized images to $256 \times 64$ sized time-series data. The hybrid model added efficacy to the work using LSTM to vanish the gradient problem and CNN to extract features automatically. On classifying the data into normal, pneumonia, and COVID-19-infected, an accuracy of $99 \%$ and PPV of $98.7 \%$ were obtained. This work also concludes that COVID-19 detection from CTs using HDNNs proves to be consistent and fast.

Other approaches that include classification of COVID-19-positive cases against COVID-19-negative cases or healthy cases [97-99] were present and also classification of COVID-19 against other pulmonary diseases as in [100], where a deep neural network with the generative adversarial network (GAN) based on synthetic data augmentation is used to classify 8 different lung pathologies. The collected dataset contains images from Digital Pathology Classification Challenge (Kaggle) and COVID-19 images from [60] comprising of 5789 atelectasis, 1010 cardiomegaly, 6331 effusion, 10317 infiltration, 6046 mass, 1971 nodule, 1062 pneumonia, 2793 pneumothorax, 84312 normal, and 337 COVID-19 images. The proposed model performed better than InceptionV3 and ResNet models with an accuracy of $89.2 \%$. Accurate lung region of interest (ROI) segmentation also takes an indispensable part in better diagnosis of COVID-19 by delineating the lesions and measuring their extent. Most of the works have used U-Net [101] architecture for this purpose $([70,71,95,102])$. U-Net is a CNN architecture developed specially for biomedical image segmentation with the ability to give both the localization information and the contextual information, which leads to the better prediction of a segmentation map. Image segmentation can also be applied to quantify the lung-infected region $([101,103,104])$, which involves visualization of the lesion distribution, prediction of severity, and assessing the progression during follow-up.

2.2. COVID-19 Prognosis. Prognosis refers to predicting the likeliness or expected disease development based on the track of the disease that is diagnosed, the condition of the patient (physical and mental), the available treatments, and other additional factors. Few COVID-19 prognosis methods are explained in this section. 
Sverzellati et al. [105] simulated the triage setting of a pandemic environment with large population of COVID19-infected suspects provided that the clinical decision should be given in the absence of any resource constraints. For this, reconstructed CXR (r-CXR) images were generated from the high-resolution CT (HRCT) images. Mortality prediction was done based on the multivariable (age, sex, duration of symptoms at triage, and a comorbidity score of $0-4$ ) by performing LR analyses to identify the contribution of clinical and radiological variables in the analysis and using a study population of 300 patients. The images were graded as follows: normal, alternative diagnosis (to be specified), indeterminate, or typical for COVID-19 pneumonia by expert radiologists. The study findings put forward that the clinicians can rely on positive CXR for showing the low or high extent of pneumonia, and in the case of the intermediate extent of CXR, it is complemented by CT for optimal stratification of high- and low-risk groups. For indicating the COVID-19 infection, the sensitivity, specificity, PPV, and NPV of HRCT are $95.2 \%, 32.8 \%, 82.2 \%$, and $67.9 \%$, respectively, which proves to be better than the corresponding metrics of r-CXR.

Wang et al. [106] proposed the COVID-19Net to identify patients of potentially high risk with poor prognosis using the transfer learning process in two steps. Initially, the network was trained on 4106 non-COVID-19 CT images from epidermal growth factor receptor (EGFR) dataset, which was then transferred to the COVID-19 dataset consisting of CT images from 1266 victims: 924 with COVID-19 (471 patients had follow-up for more than 5 days) and 342 with other pneumonia. For prognostic analysis, 64-dimensional DL features were combined with clinical features (age, sex, and comorbidity) to compose an integrated feature vector. Then, a multivariate Cox proportional hazard model was used to predict the risk of a patient. The Kaplan-Meier analysis and log-rank test implied that the deep features have promising prognostic value for COVID-19 $(p<0.0001$, $p=0.013$, and $p=0.014$ in 3 datasets).

Feng et al. in [107] explored the predictive value of COVID-19 prognosis from chest CT images by comparing the difference in clinical and CT characteristics in the progressive and stable patients by performing multivariate LR and nomogram establishment. Older age, CT severity score on admission, and higher neutrophil-to-lymphocyte ratio (NLR) were identified to be the independent and significant predictive aspects for advancement to severe COVID-19 infection during hospitalization and were supported by an appreciative calibration of the nomogram, a nonsignificant Hosmer-Lemeshow test statistic $(p=0.791)$, and AUC value of 0.898 in the validation cohort. This method was simple with only three easily obtainable variables and was capable of promptly predicting the progression risk (in-hospital) in the moderate stage of COVID-19 patients within 14 days. It was performed on an unbalanced data consisting of only $10 \%$ of patients developing severe COVID-19 pneumonia, which seems to be a limitation of this work.

Liang et al. [108] developed and validated a risk prediction method for early diagnosis of COVID-19 infection in patients. For this, different clinical, laboratory, epidemiological, and radiological image variables were screened at the time of admission in medical center/hospital to predict the risk score as low-, moderate-, and high-risk cases. The Least Absolute Shrinkage and Selection Operator (LASSO) was used for screening the variables, and LR was used to formulate the predictive risk score (COVID-GRAM). This method was developed with a cohort of 1590 patients and validated on 710 patients to estimate the risk that they will develop a critical illness. 10 variables including chest radiographic abnormality, age, cancer history, number of comorbidities, and NLR were identified as independent predictive factors among 72 potential factors by the LR model. A mean accuracy of 0.88 was obtained in the validation group. This was designed as a Web-based calculator to assist the clinicians in estimating the possibility of developing critical ailment in individual hospitalized victims. As the development and validation patient group was completely selected from a particular county, there might be limitation in generalizing the work for patients from different regions.

Wu et al. [109] used CT images to develop an easy-to-use and noninvasive prognosis method to predict the clinical risk of COVID-19 patient outcome as death, need for mechanical ventilation, and admission to the intensive care unit. The development cohort consists of 492 patients grouped into the early-phase group and the late-phase group based on their CT scanning performed one week before or after the symptom onset, respectively. A fine-gray competing risk regression model was used to frame the clinical model and CrrScore (the clinic-radiomic signature), and a Least Absolute Shrinkage and Selection Operator (LASSO) was used to construct the RadScore (the radiomic signature). In the late-phase group, the radiomic signature alone proved to be efficient to forecast the poor outcome in patients with an AUC value of 0.976 and C-index of 0.885 . In the case of the early-phase group, the clinic-radiomic signature exhibited better efficacy with an AUC value of 0.862 and C-index of 0.850. Therefore, based on the time of CT scanning concerning the symptom onset, appropriate signatures can be used for predicting the prognostic outcome.

Research works on the prognostic analysis of COVID-19 using radiological images are minimal and need to be further explored to keep a check on the severity of the diseases and reduce the mortality rate. For prognosis, the clinical features are combined with the radiological image findings to predict the patient's medical condition for delivering successful triage and lessen the disease spread.

2.3. COVID-19 Severity/Risk Detection. A streamlined severity/risk detection mechanism is highly required for COVID-19 triage to lower the prodigious rate of mortality. Apart from early screening, the severity assessment also plays a vital role in triage and disease management. A review on the related works in the literature is discussed below.

Cohen et al. [110] built a severity prediction model to assist the clinicians in managing the patient care using a regression model to predict two types of scores: extent of 
lung involvement (0-8 score) by ground-glass opacity or consolidation and degree of opacity (0-6 score) on the COVID-19-infected CXR images. DenseNet was employed to predict pneumonia from 94 COVID-19 CXR images acquired from [8]. The model was trained with 7 datasets [90, 98, 111-115] with 18 common radiological finding tasks consisting of 88,079 non-COVID-19 CXR images. Just using a single feature (lung opacity) for risk predictions countered to the ground truth value of prediction score, the model was capable of better prediction in both the opacity score and the geographic extent of infection. The Pearson correlation coefficient and $R^{2}$ for lung opacity score prediction task are $0.78 \pm 0.04$ and $0.58 \pm 0.09$, respectively. Similarly, for the geographic extent prediction the Pearson correlation coefficient of $0.80 \pm 0.05$ and $R^{2}$ value of $0.60 \pm 0.09$ were obtained. It was capable of predicting the geographic extent score (range 0-8) with 1.14 mean absolute error (MAE) and lung opacity score (range $0-6$ ) with 0.78 MAE generalization that can be improved by performing large-scale evaluations on public datasets from around the world.

Zhu et al. [116] also employed a model similar to [110] for accurate staging of COVID-19 severity on CXRs. A deep CNN model was used to foresee the lung severity scores from 131 CXRs based on the degree of opacity (0-3 score) and geographic extent (0-4 score). A correlation analysis was performed amidst the predicted score and the radiologist scores, which resulted in a higher value of 0.90 and a MAE of $8.5 \%$, making the model yield top results. An average opacity score of 2.52 and average geographic extent score of 3.42 were obtained across three readers.

Tang et al. [17] proposed a severity assessment model to categorize the COVID-19-infected CT images as severe or non-severe. For these, 63 quantitative features of top importance such as volume and ratio of the left/right/whole lung and volume of GGO were extracted from 176 CT images obtained from different hospitals (using different scanners) and trained to the random forest model. An accuracy of 0.85 , AUC value of 0.91 , true positive rate of 0.933 , and true negative rate of 0.745 were obtained for this model. The volume and ratio of GGO were identified to be the feature with most importance to estimate the severity of the disease, and another finding from the study revealed that the quantitative features observed in the right lung were more significantly related to COVID-19 severity than the features of the left lung. The main drawback is that the model is able to label the images as only severe or non-severe instead of multiple classifications such as mild, common, severe, and critical.

The limitation of [17] can be overcome by the COVIDSDNet proposed by Tabik et al. [117], which has better generalization capability. A balanced and homogeneous database, COVIDGR-1.0, was built, which includes different levels of severity such as normal with positive RT-PCR (normal PCR+), mild, moderate, and severe. It consists of 426 COVID-19-positive CXR images and 426 COVID-19negative images (normal PCR: 76, mild: 100, moderate: 171, and severe: 79). It performs smart data generation using a class-inherent transformation approach motivated by GAN and ResNet-50 loaded with ImageNet weights for classification. Better and more stable results and great balance between specificity and sensitivity were obtained. Comparing the classification accuracies of 4-class classification (normal PCR +: $28.42 \% \pm 2.58$, mild: $61.80 \% \pm 5.49$, moderate: $86.90 \% \pm 3.20$, and severe: $97.72 \% \pm 0.95)$ and 3class classification (mild: 46.00\% \pm 7.10 , moderate: $85.38 \% \pm 1.85$, and severe: $97.22 \% \pm 1.86$ ), even though normal PCR + seems to be the toughest level to predict, its existence accelerates the accuracy of the minor severity levels, notably mild level. It is also observed that the segmentation of the lung region using U-Net has essentially improved the sensitivity value.

Severity assessment in COVID-19 mostly relies on classifying the pre-identified radiological images or using the clinical data of patients to perform the severity analysis, but pre-identification of radiological images as mild, moderate, or severe infections may be challenging and difficult.

2.4. Inferences. Based on the study described in the previous sections, the review findings and inferences are listed below:

(i) COVID-19 diagnosis can be performed by classification or segmenting the infected region. Classification can be viable and easy to implement in short time as it demands only weak image-level labels and few model specifications for training the classification model.

(ii) Classification of COVID-19 infection against normal cases seems to be much easier with high classification results and performance metrics. In general, binary classification (COVID-19/nonCOVID-19, COVID-19/other pneumonia) yields better results than multiclass classification (COVID-19/other pneumonia/normal/other lung diseases).

(iii) Distinguishing COVID-19 from other pneumonia, especially viral pneumonia, is challenging as they show similar characteristics in the radiological images. In such cases, the efficiency of the classifier can be improved by adding more images or other types so that the learning process can be enhanced during training. The performance of distinguishing COVID-19 from normal or bacterial pneumonia can yield better results since there is significant variation in the radiological image features.

(iv) Deep Learning methods are mostly preferred than the machine learning methods for feature extraction as they can extract the inherent deep features specific to each class for a finer classification. The most commonly used deep models for feature extraction and classification that give promising results are DenseNet, ResNet, VGG, and their modified variants. Other networks like Inception, Exception, ShuffleNet, and EfficientNet also have been used in many works. The CNN layer implementation with residual connection is depicted in Figure 6. Diagnosis of COVID-19 employing deep learning techniques have shown 


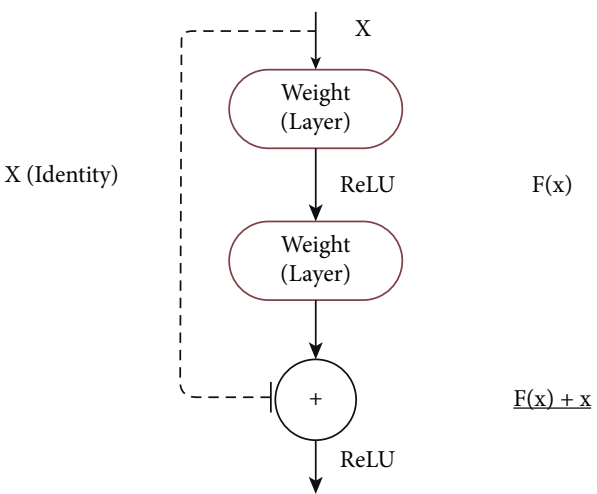

Figure 6: Sample residual connection used in ResNet [40].

better sensitivity and specificity than the radiologist's decision. U-Net is the most widely preferred deep learning architecture for segmentation task, which is depicted in Figure 7.

(v) Deep features can be combined with clinical data such as clinical symptoms, nucleic acid detection results, epidemiology, and laboratory indicators, which can bypass any misdiagnosis and efficaciously improve the clinical triage.

(vi) Prognosis of COVID-19 is of equal importance as that of diagnosis, since it demands medical triage and management of the patient care. Early identification of the disease can aid in the diagnostic ambiguity of radiologists. Works on COVID-19 prognosis are minimal, so this can open up a large research path for many researchers.

(vii) Prediction of COVID-19 infection severity plays a vital role in making clinical decisions so that the medical team can work towards reducing the mortality rate.

(viii) Despite CXRs being cheap and easily obtainable, CTs are highly preferred for COVID-19 analysis as they are capable of early detection of the disease even in victims with negative RT-PCR tests, in asymptotic patients or even ahead symptoms may arise.

Figure 8 illustrates the inferences from the review of different tasks related to medical image analysis of COVID19.

\section{Related Reviews in the Field}

There are have been previous reviews $[52,118]$ that have encompassed most of the research regarding COVID-19 in terms of machine learning, deep learning, and medical imaging along with its analysis and scrutinized them to preference inferences, to promote further research in the field. They also present challenges that future researchers should tackle to incorporate better results and build more efficient models. Table 6 (while there are other reviews present, they were either extremely short, or did not contain valuable information, or were mostly covered in the mentioned reviews.) lists out the most useful reviews, which have taken place till date and their respective merits and limitations. Most reviews covered the architectures used quite broadly and have also made studies in context to their usage (pre-trained or incorporation for custom methods). A general pipeline of the same is shown in Figure 9. Another aspect that was covered in multiple reviews was the use and availability of public datasets, which is paramount to expand the COVID-19 research capabilities.

Model generalization has also been tackled in numerous studies as it is an important aspect to be considered while building deep learning-based models. While the reviews have covered the majority of the research taking place and the challenges accompanied by them, only Shorten et al. [52] accounted for extending work via privacy-preserving methods and mentioned research taking place through other deep learning paradigms such as meta-learning [126] and self-supervised learning [127]. Apart from these, the readmission risk of COVID-19-recovered patients can also be analyzed using a predictive model. Many ML- and DL-based predictive models have been designed to predict the readmission risk of patients discharged from hospitals for various diseases $[128,129]$. Increased readmission rates may be liable to high healthcare cost and risk of inpatient hospital mortalities. Several works have been carried out to improve the performance of these predictive models using evaluation metrics [130, 131]. Similarly, many studies have been conducted regarding the COVID-19 case readmission rates and factors $[132,133]$.

In the field of medicine, data privacy is of utmost importance and is always the leading cause for the shortage of open-source data. Addressing this issue should be the first among the list of challenges concerning COVID-19. One of the main reasons for having such expansive development and testing is because of the large amounts of open-source data present (including open accessing all research), which is generally absent for other diseases. Self-supervised learning approaches have proven to surpass the usual supervised deep learning methods in $[134,135]$ and should be given more importance and consideration when topics of extension and challenges are brought upon. There is also a major gap in accounting for the research conducted in terms of prognosis for COVID-19. There is no single review that focuses on this aspect. In terms of medical image analysis, few solutions are addressed to the challenges mentioned in [52]. To the best of our knowledge, the previous reviews missed to cover the topics discussed above. Additional information is all part of recent developments, which have taken place post the drafting of those reviews.

3.1. Extension of Discussion on the Limitations of Deep Learning Approaches Discussed in Shorten et al. (2020) [52]

(a) Explainability: deep learning models are often called black box models due to their non-interpretive behavior. This highly disregards using deep learning models on sensitive real-world tasks such as medical image analysis and has hence turned into a nontrivial 


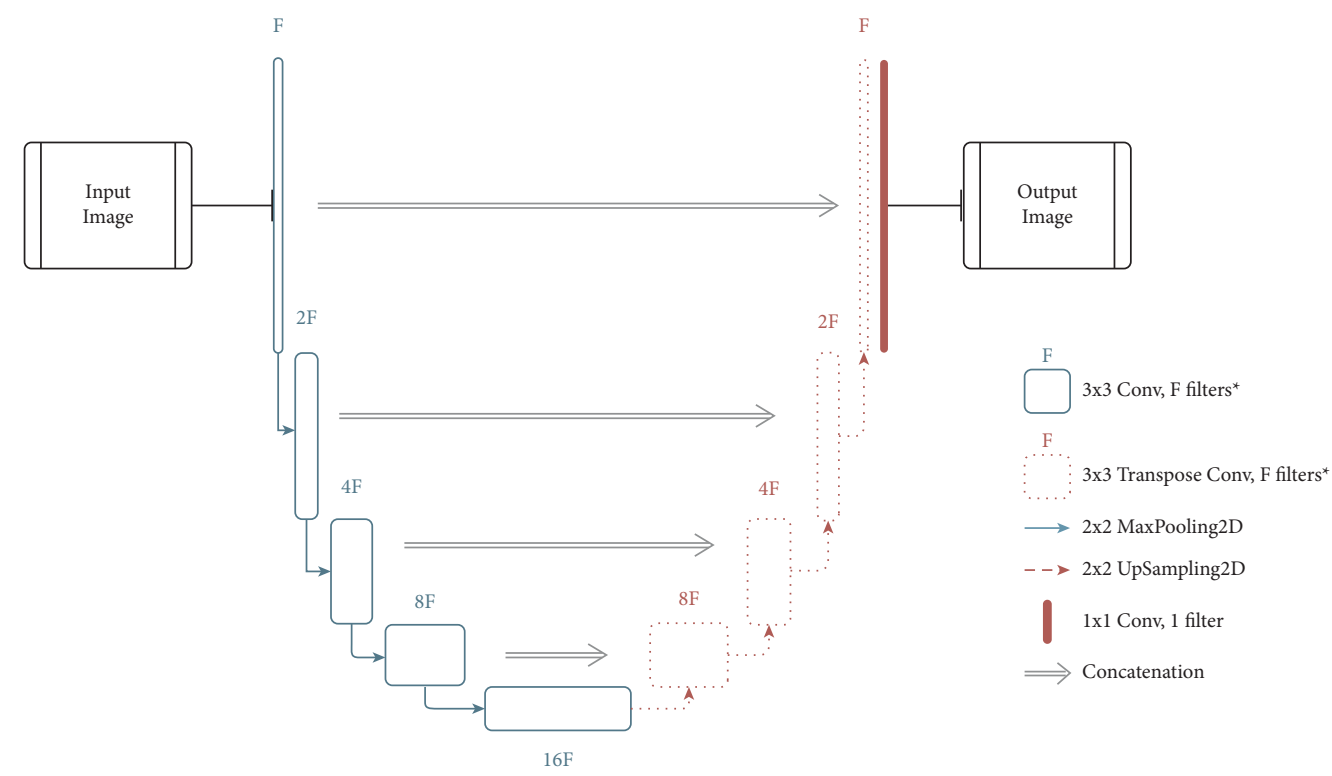

Figure 7: Sample U-Net architecture for medical image segmentation. * in the legend indicates that the filter is followed by a batch normalization layer and a ReLU function.
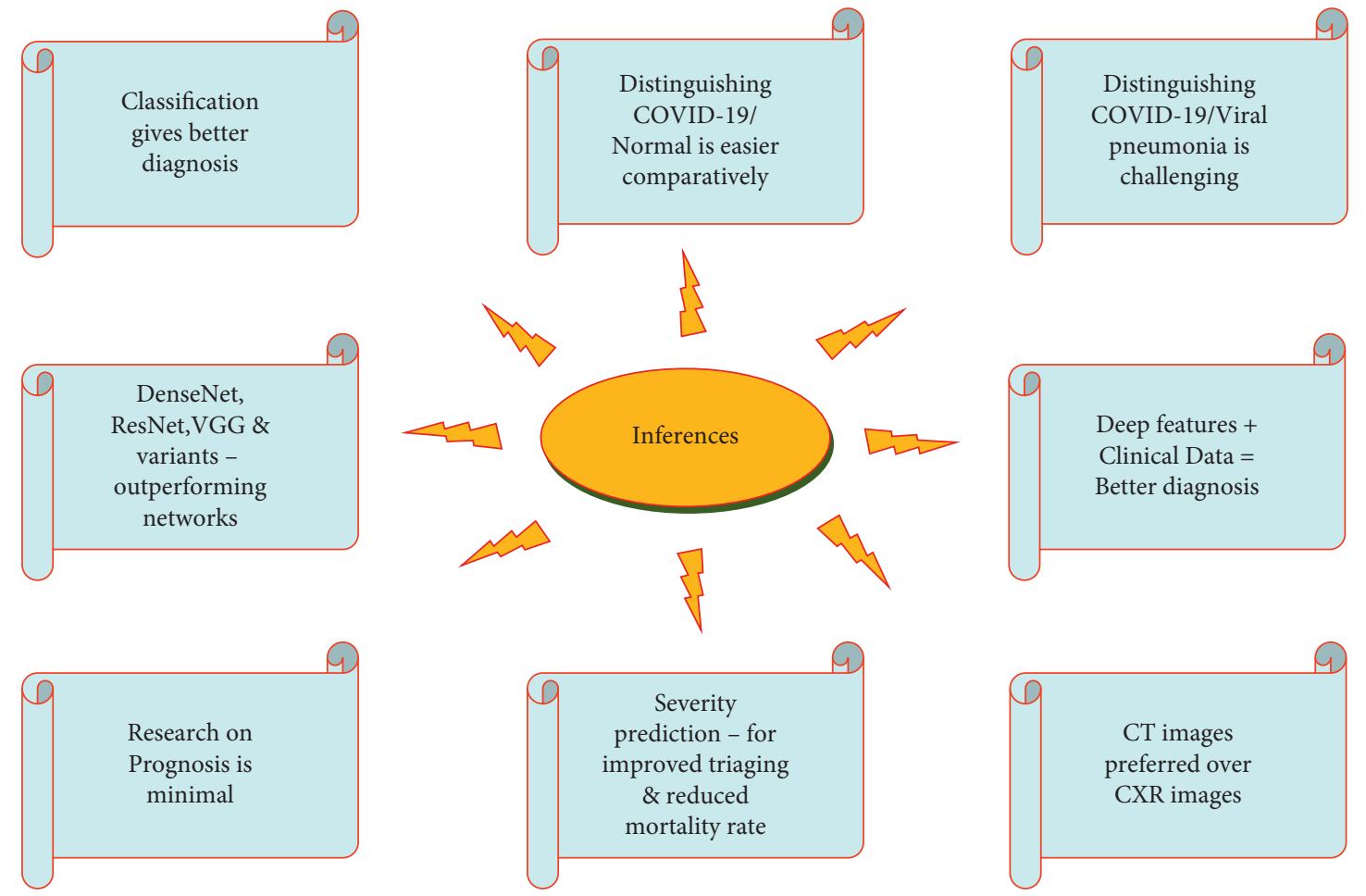

FIGURE 8: Inferences from the review of COVID-19 medical image analysis.

issue. With the focus here being the same, there are ample explainable techniques that have come up to aid in explaining vision-based deep learning models. Table 7 shows the current state-of-the-art methods used to help interpret vision-based deep learning models. Score-CAM eliminates the dependence on gradients (as seen in Grad-CAM) by securing the weight of individual activation maps, by virtue of its forward passing score on the aimed target class, which results in a linear combination of the activation maps and weights. EVET [135] proposes a heuristic pipeline for strengthening the visual explanations by applying image transformations. Explainability in segmentation tasks (primarily done by U-Nets) is a field that is still being heavily explored. Initial attempts have been done by adapting 
TABLE 6: Merits and limitations of existing review papers exploring the broad depth of COVID-19 research in terms of medical imaging, medical image analysis, machine learning, and deep learning.

\begin{tabular}{|c|c|c|}
\hline Revi & Merits & Limitations \\
\hline $\begin{array}{l}\text { Ozsahin et al. } \\
\text { [119] }\end{array}$ & Classified different groups of studies. & $\begin{array}{l}\text { Only highlights result and techniques without any } \\
\text { intuition as to why either are used. } \\
\text { Includes segmentation models within classification } \\
\text { studies. }\end{array}$ \\
\hline $\begin{array}{l}\text { Shoeibi et al. } \\
\text { [120] }\end{array}$ & $\begin{array}{l}\text { Includes a forecasting study of coronavirus prevalence in } \\
\text { multiple countries. } \\
\text { Includes pre- and post-processing techniques used in various } \\
\text { COVID-19 detection approaches. }\end{array}$ & $\begin{array}{l}\text { Certain figures depict subpar comparisons and include } \\
\text { unnecessary comparison samples. } \\
\text { The review is more focused on architectures utilized } \\
\text { rather than the inference generated from the literature. }\end{array}$ \\
\hline Pham | & $\begin{array}{r}\text { Presents many stron } \\
\text { Alleviates the task of } \\
\text { Dens }\end{array}$ & $\begin{array}{c}\text { Should have considered the use of the Matthews } \\
\text { correlation coefficient (MCC) [121] as binary } \\
\text { classification was considered. }\end{array}$ \\
\hline Shor & $\begin{array}{r}\text { Pinpoints key disc } \\
\text { approaches and the } \\
\text { domains a }\end{array}$ & $\begin{array}{l}\text { Falsely claims the first paper to re } \\
\text { point of view for COVI }\end{array}$ \\
\hline & $\begin{array}{r}\text { Explores several supporting domains } \\
\text { learning, meta-learning, and self-supervise } \\
\text { missed in most review }\end{array}$ & $\begin{array}{r}\text { Compares paper to other "artifici } \\
\text { methods to their ap }\end{array}$ \\
\hline $\begin{array}{l}\text { Alsharif et al. } \\
{[122]}\end{array}$ & $\begin{array}{l}\text { Attempts to compare deep learning to machine learning } \\
\text { approaches. } \\
\text { The review is inclined to help beginners in the field. }\end{array}$ & $\begin{array}{l}\text { Fails to dive } \\
\text { ince }\end{array}$ \\
\hline Joy e & $\begin{array}{r}\text { It poses an extensive study } \mathrm{c} \\
\text { archit }\end{array}$ & No challenges are mentioned or analyzed. \\
\hline $\begin{array}{l}\text { Alghamdi } \\
\text { et al. [124] }\end{array}$ & $\begin{array}{c}\text { Gives in-depth analysis about architectures and the various } \\
\text { constraints in tandem to them such as data, explainability, and } \\
\text { more. }\end{array}$ & $\begin{array}{c}\text { Does not consider the SOTA methods in explainability } \\
\text { terms. } \\
\text { Fails to address other possible learning paradigms and } \\
\text { privacy-preserving methods. } \\
\text { Should be mentioned as the review is architecture- } \\
\text { dominated. }\end{array}$ \\
\hline $\begin{array}{l}\text { Islam et al. } \\
{[125]}\end{array}$ & $\begin{array}{l}\text { Gives an extensive study on open challenges. } \\
\text { Highlights the data partitioning techniques. }\end{array}$ & nitations are \\
\hline
\end{tabular}

${ }^{1}$ While there are other reviews present, they were either extremely short, or did not contain valuable information, or were mostly covered in the mentioned reviews.

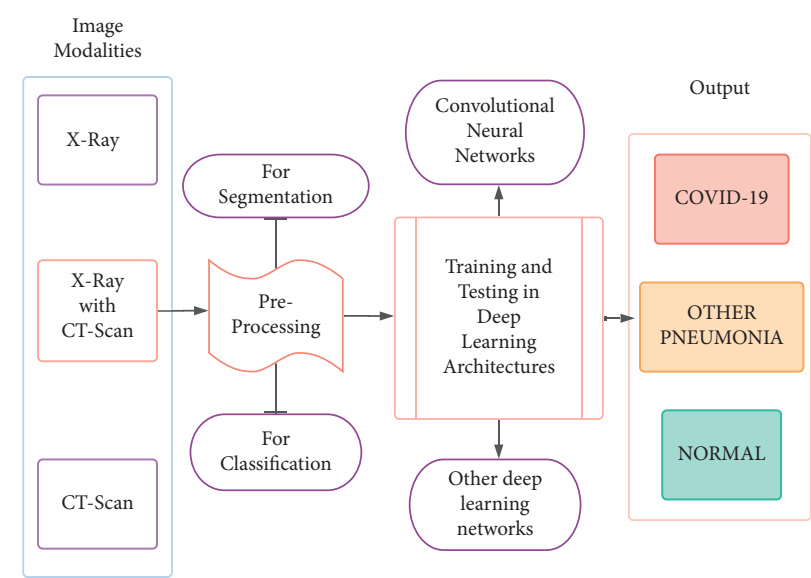

FIGURE 9: Generalized pipeline of COVID-19 detection from radiological image modalities. 
TABLE 7: State-of-the-art explainable techniques for vision-based deep learning models.

\begin{tabular}{lc}
\hline Task & Explainable method \\
\hline & Grad-CAM [48] \\
Classification & Score-CAM [49] \\
& EVET [135] \\
Segmentation & SEG-GRAD-CAM [136] \\
\hline
\end{tabular}

Grad-CAM to segmentation in the form of SEGGRAD-CAM [136]. The origin of the above work comes from [137]. Explainable models can benefit the medical image analysis pipeline in many ways. It helps understand where the model is focusing on the image, increase user confidence, and inspect the model at a deeper level, which in turn helps in debugging the model as well.

(b) Generalization Metrics: precision is generally the major metric taken into consideration while accounting for a fair metric for medical image classification methods. While considering segmentation, the authors in [138] give a detailed description regarding which metrics to consider. They also mention the use of precision here as well. A detailed study on generalization concepts and metrics can also be studied in [139].

(c) Learning From Limited Labeled Datasets or Unlabeled Data: primary focus on two paradigms of learning is as follows:

(i) Meta-Learning: it follows the approach of learning to learn. It is used to adapt to learn new environments and in a quicker fashion greatly aligns with the demand of COVID-19 research. It also requires lesser data samples. In [118], a trainable $\mathrm{n}$-shot deep meta-learning framework was built to classify COVID-19 cases with limited training CXR images. Another aspect of meta-learning is neural architecture search (NAS) and that has been observed to work better than many baseline models [140].

(ii) Self-Supervised Learning: it is a subgroup of unsupervised learning, which works on the basis of training the deep learning model explicitly with automatically generated labels. As Figure 10 depicts, the process involves learning visual features from pretext task (tasks predesigned for networks to deal with) and acts as a pre-trained model for other downstream tasks (computer vision applications to examine the self-supervised learned feature quality) via fine-tuning. References $[133,134]$ have rivaled the top-performing models in image tasks, even surpassing the supervised methods. Reference [141] showed that the combination of data augmentation and self-supervised learning has outperformed all previous approaches in severity assessment.

(d) Data Privacy: a detailed discussion of data privacy is given in [52]. To extend on the avenues mentioned

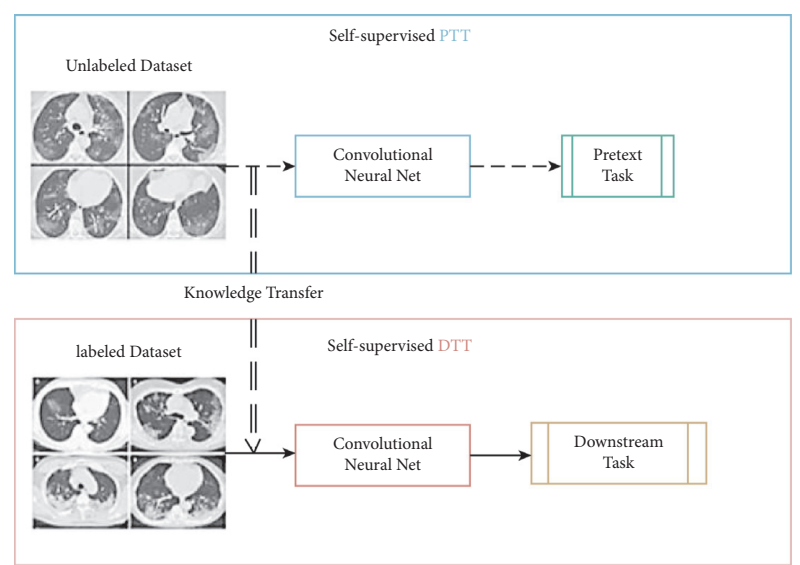

FIGURE 10: General self-supervised learning pipeline. PTT: pretext task training; DTT: downstream task training.

there, the use of differentially private federated neural architecture search [142] is recommended to preserve data privacy. Through this method, a model can be tested on several subsets of data, which contain varied distributions and distinctions from other datasets and in parallel keep any information about the various data samples completely privatized. Although the method is very computationally demanding, it can help screen through different samples of data and greatly test the robustness of any model. Figure 11 depicts the working of both NAS and federated NAS (FNAS) [143]. An application of federated learning in terms of prognosis can be seen in [144]. A noise implementation algorithm is integrated with a cross-device federated learning, such that the initial symptom prognosis can be achieved during a pandemic like COVID-19.

\section{Pre- and Post-Processing Techniques for COVID-19 Medical Image Analysis}

4.1. Preprocessing. Preprocessing is a crucial part of vision models' pipeline. The process involves performing operations at the lowest level of abstraction. The objective is to enhance the picture information that suppresses undesired deformities or improves the image features necessary for continued transformations, which is mainly linked with generating higher accuracy in models. Even simple techniques such as resizing or cropping the image can make major difference in deep learning models. For example, cropping out the redundant parts of a scan can help the deep learning model avoid unnecessarily parsing through that spatial information to concentrate on the more essential areas of the scan. Certain models require specific size of input images to fit in. In such situations, rescaling the image is completely unavoidable.

Figure 12 depicts a chest CT scan being put through contrast-limited adaptive histogram equalization (CLAHE) in comparison with an original CT image. A clear depiction of sharper visual features after CLAHE is applied, which makes it easier for the model to develop and correlate these 


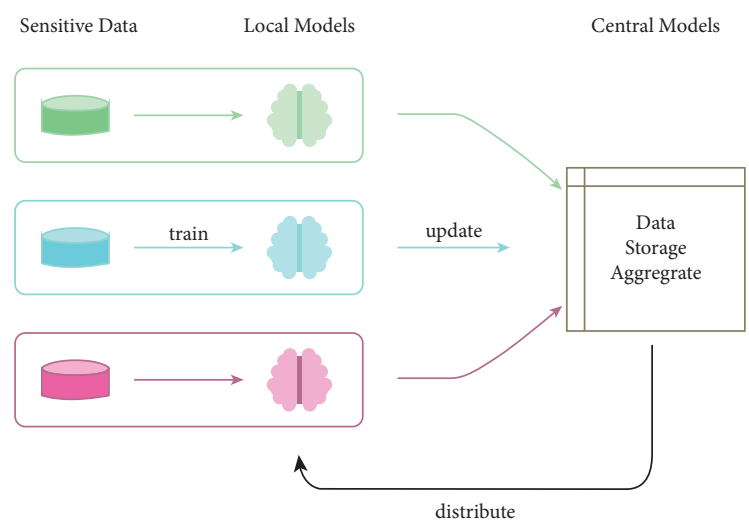

Figure 11: General federated learning pipeline.
ORIGINAL
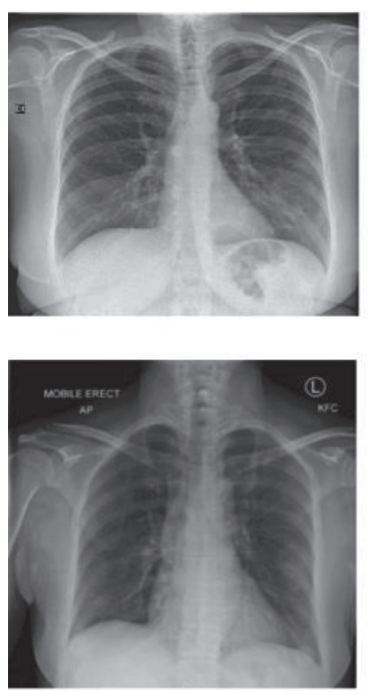

CLAHE
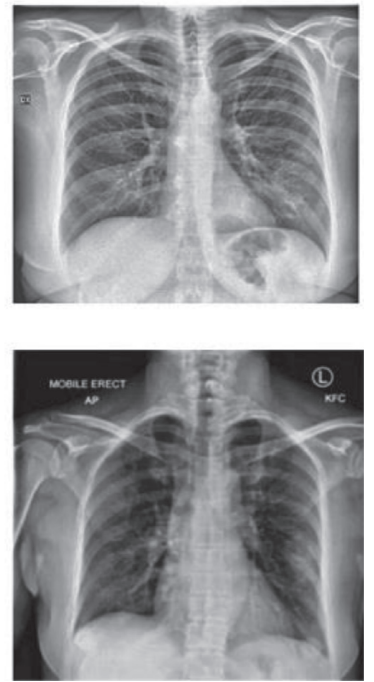

FIgURE 12: Original CT image versus CLAHE-processed CT image [145].

visual cues. Figure 13 illustrates how the preprocessing step can also help better express the image features through image enlargement. The enlarged points are first targeted (shown as question marks) and then filled through interpolation. A comparative image is also shown with no interpolation done. In addition to the techniques mentioned in Table 8, there are certain methods that can aid in preprocessing. Data augmentation is a widely used method in much literature to help increase the training sample size. GANs have also been applied to increase the sample size [153]. Noise removal techniques without losing the significant edges can also be used to enhance the images [154].

4.2. Post-Processing. Post-processing generally directs to improvement in the images after the model has given an output, but in the case of medical image analysis, it mainly involves generating inferences from the model outputs via explainability measures. The basis of most techniques is class activation maps [137]. These methods are used to pinpoint the focus of the model and understand whether the output generated is on the basis of the detection of the actual disease and not any other factors. The extensions made to [137] are discussed in Section 3.1.1. In [155], a method called the Peekaboo training scheme was used, in which a two-stage patch crop-and-drop strategy promotes the model to furnish activation maps for every target concept.

\section{Discussion}

In this section, we discuss additional challenges faced while conducting experiments and how the work done with respect to COVID-19 can help the field of medical image analysis in general.

5.1. Challenges Faced. Reviewing of multiple literature samples led to the identification of multiple challenges present in the domain, a few of which are already covered in Section 3.1. In this section, another set of challenges that have been discovered is elucidated.

(i) Interclass analogy and intraclass deviation of pneumonia lesions: COVID-19 pneumonia, which is also caused by viral infection, contains indicative overlay of features and radiological image characteristics with other viral pneumonia leading to the interclass analogical problem. Another problem that arises while dealing with the pulmonary medical images is the intensity in-homogeneity problem caused by the closeness of gray level between the different soft tissues, resulting in segmentation and detection difficulties [156]. Detecting the anomalous features from the medical images becomes challenging due to the noise impedances from the tissues and lesions. The infected region may still contain some non-lesion regions with wide variations in tissues, which further makes it complicated to differentiate.

(ii) Generalization and reproducibility: the COVID-19 detection algorithms proposed by various researchers produce great results for the particular small dataset used in that work. When these trained classifier algorithms are implemented on larger unseen data, they may not be able to generalize their performance. Moreover, problems arise in reproducing the similar performance on other multicenter datasets. One such solution to this problem is the use of vision transformers, which have demonstrated superior performance and greater generalization prowess. These are of paramount importance in the context of a deployment scenario [157].

(iii) Data source learning problem of ML and DL: on applying neural network-based COVID-19 detection protocols to multicenter datasets, most of the detection systems tend to learn the source of dataset, their imaging protocols, mode, and so on, rather than learning the discriminative features among the various classes. Such kind of algorithms may not be 


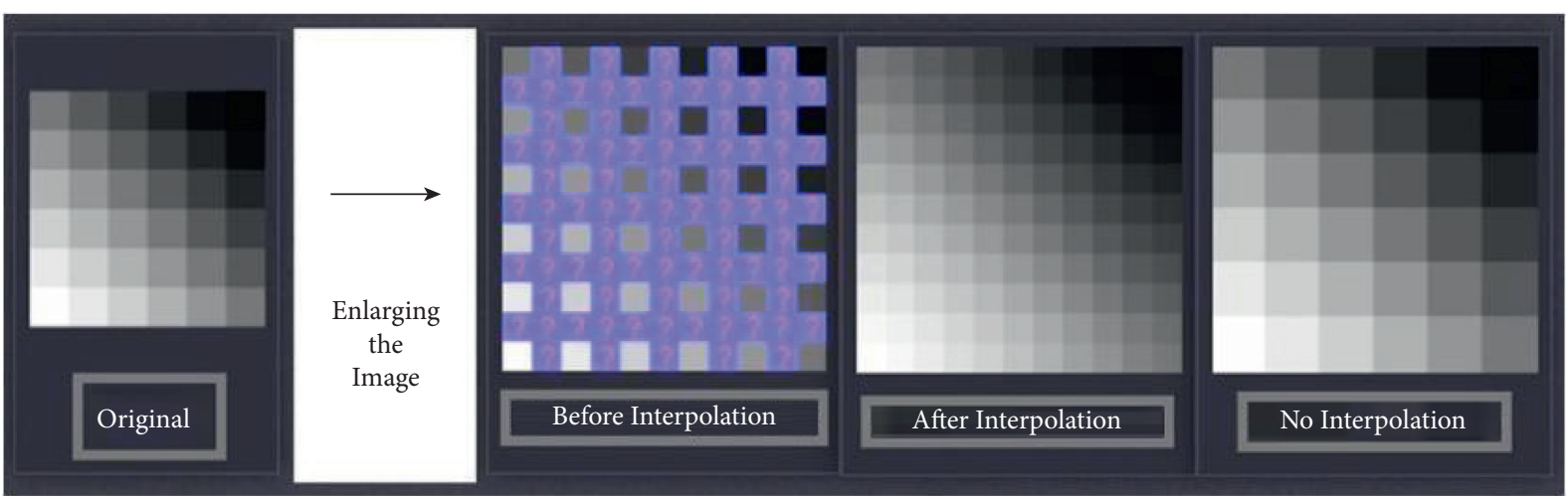

FIGURE 13: Depiction of a pipeline for enlarging an image through interpolation.

TABLE 8: List of preprocessing techniques used for analyzing radiological images.

\begin{tabular}{|c|c|c|}
\hline Reference & Technique & Utilization \\
\hline Pizer et al. [146] & $\begin{array}{l}\text { Adaptive histogram } \\
\text { equalization }\end{array}$ & Improves contrasts in images. \\
\hline $\begin{array}{l}\text { Veldhuizen and Jernigan } \\
\text { [147] }\end{array}$ & Wiener filter & Produces an estimate of a desired or target random process. \\
\hline Lehmann et al. [148] & Interpolation & $\begin{array}{c}\text { Best estimation of a pixel's color and intensity in context to the values at } \\
\text { neighboring pixels. }\end{array}$ \\
\hline Tian et al. [149] & Binarization & Transforms data features of any entity into vectors of binary numbers. \\
\hline Yadav et al. [145] & CLAHE & $\begin{array}{l}\text { Amplifies the contrasts. } \\
\text { Works on small regions called tiles. }\end{array}$ \\
\hline Prabha and Kumar [150] & Smoothing filter & Utilized in blurring regions. \\
\hline Kociołek et al. [151] & Normalization & Changes the range of pixel intensity values. \\
\hline Gungor [152] & Wavelet transform & $\begin{array}{l}\text { Reduces noise in images. } \\
\text { Decomposes special patterns hidden in mass of data. }\end{array}$ \\
\hline
\end{tabular}

fair enough when generalized for different data [158].

(iv) Spread and contamination: contamination of the scanners is also an issue that needs to be considered. There is a great possibility of disease spread during scanning; hence, the radiologists must assure that the scanners are maintained clean after every scanning process.

5.2. Future Scope: Utilizing COVID-19 Medical Image Analysis Research in Other Fields. As seen, enormous amounts of effort take place to furnish new methods through deep learning strategy to tackle the problems of COVID-19 detection. A few potential avenues have been mentioned in this section, which can in general help to extend the field of medical image analysis.

(i) Medical model weights and baseline architectures: utilizing model weights built upon disease-affected scans has shown to improve the efficiency in models [87]. Particular to COVID-19, CheXNet [159] weights utilized in models instead of the usual ImageNet weights have been shown to increase the model [160], which can be utilized to detect other diseases as well. General vision problems are quite different when compared to dealing with medical images such as MRIs, CTs, and PET scans. Training models that are based on the weights gained from training on such images should furnish more accurate models. Apart from COVID-19, there are many other diseases that can be detected from radiographs. As DenseNet is seen to do well for COVID-19 (both from pre-training and for customized models), it should be fair enough for other diseases as well and can act as a good starting point for future researchers to expand existing works.

(ii) Expanding the community and model testing: there are many introductory materials focused on COVID-19 and medical image analysis. This can help build the community further and attract novices to the field. Testing models on scarce datasets make it quite difficult to generate inference about the model's performance. With the massive open-source data available, it also allows potential researchers to realistically test their models and architectures. The variety of data present also helps in testing the model for generalizability, which is one of the biggest obstacles to surmount while utilizing deep learningbased methodologies.

\section{Conclusion}

A comprehensive description on the various COVID-19 detection techniques using medical image analysis has been 
described in this study. The cause, effect, challenges, limitations, and other retrospective discussions on COVID-19 medical image analysis have been discussed through this study to best feature the importance of carrying out more research on this area to reduce the increased mortality count faced by the world. DL can improve the disease diagnosis efficiency by precisely locating the infections in the medical images in a faster and accurate manner. The preceding COVID-19 analysis methodologies proposed by various researchers can be used only as a reinforcement technique to assist the medical teams in highly populated areas and in situations requiring quicker diagnosis. The problems such as unavailability of enough and accurate labeled data, generalization and reproducibility of preceding algorithms for multicenter large datasets, and difficulty to isolate COVID19 against other pneumonia cases due to the closeness of gray level of the soft lung tissues have been highly challenging to design a diagnostic system with high reliability and accuracy. An intelligent and accurate computer-assisted COVID-19 diagnostic system employing adaptive deep learning models with active/incremental learning is the need of the hour to combat the evolving coronavirus.

\section{Data Availability}

The data supporting this systematic review are from previously reported studies and datasets, which have been cited.

\section{Conflicts of Interest}

The authors declare that they have no conflicts of interest.

\section{Acknowledgments}

This work was supported by the 2020 EBC-C (Extra-Budgetary Contributions from China) Project on Promoting the Use of ICT for Achievement of Sustainable Development Goals and University Malaya under Grant IF015-2021.

\section{References}

[1] W. Wang, Y. Xu, R. Gao et al., "Detection of SARS-CoV-2 in different types of clinical specimens," JAMA, vol. 323, pp. 1843-1844, 2020.

[2] T. C. Kwee and R. M. Kwee, "Chest CT in COVID-19: what the radiologist needs to know," RadioGraphics, vol. 40, no. 7, pp. 1848-1865, 2020.

[3] C. P. West, V. M. Montori, and P. Sampathkumar, "COVID19 testing," Mayo Clinic Proceedings, vol. 95, no. 6, pp. 1127-1129, 2020.

[4] M. Y. Ng, E. Y. P. Lee, J. Yang et al., "Imaging profile of the COVID-19 infection: radiologic findings and literature review," Radiology. Cardiothoracic imaging, vol. 2, no. 1, p. e200034, Article ID e200034, 2020.

[5] C. Huang, Y. Wang, X. Li et al., "Clinical features of patients infected with 2019 novel coronavirus in Wuhan, China," The Lancet, vol. 395, no. 10223, pp. 497-506, 2020.

[6] M. Durrani, I. U Haq, U. Kalsoom, and A Yousaf, "Chest $\mathrm{X}$-rays findings in COVID-19 patients at a University Teaching Hospital - a descriptive study," Pakistan journal of medical sciences, vol. 36, p. S22, 2020.
[7] M. Emmanuel, C. Milena, C. C. Alexandre et al., "Deep learning workflow in radiology: a primer," Insights Imaging, vol. 11, 2020.

[8] D. R. Sarvamangala and R. V. Kulkarni, "Convolutional neural networks in medical image understanding: a survey," Evolutionary intelligence, pp. 1-22, 2021.

[9] S. Soffer, A. Ben-Cohen, O. Shimon, M. M. Amitai, H. Greenspan, and E. Klang, "Convolutional neural networks for radiologic images: a radiologist's guide," Radiology, vol. 290, no. 3, pp. 590-606, 2019.

[10] N. Sharma, V. Jain, and A. Mishra, "An analysis of convolutional neural networks for image classification," Procedia Computer Science, vol. 132, pp. 377-384, 2018.

[11] H.-C. Shin, H. R. Roth, M. Gao et al., "Deep convolutional neural networks for computer-aided detection: CNN architectures, dataset characteristics and transfer learning," IEEE Transactions on Medical Imaging, vol. 35, no. 5, pp. 1285-1298, 2016.

[12] S. Minaee, Y. Y. Boykov, F. Porikli, A. J. Plaza, N. Kehtarnavaz, and D. Terzopoulos, "Image segmentation using deep learning: a survey," IEEE Transactions on Pattern Analysis and Machine Intelligence, 2021.

[13] N. Srivastava, G. Hinton, A. Krizhevsky, I. Sutskever, and R. Salakhutdinov, "Dropout: a simple way to prevent neural networks from overfitting," Journal of Machine Learning Research, vol. 15, pp. 1929-1958, 2014.

[14] S. J. Pan and Q. Yang, "A survey on transfer learning," IEEE Transactions on Knowledge and Data Engineering, vol. 22, pp. 1345-1359, 2009.

[15] S. Ioffe and C. Szegedy, Batch Normalization: Accelerating Deep Network Training by Reducing Internal Covariate Shift, pp. 448-456, PMLR, New York City, NY, USA, 2015.

[16] Y. Wu and K. He, "Group normalization," Computer Vision ECCV 2018, pp. 3-19, 2018.

[17] Z. Tang, W. Zhao, X. Xie et al., "Severity assessment of coronavirus disease 2019 (COVID-19) using quantitative features from chest CT images," 2020, https://arxiv.org/abs/ 2003.11988

[18] H. Yasar and M. Ceylan, "A novel comparative study for detection of COVID-19 on CT lung images using texture analysis, machine learning, and deep learning methods," Multimedia Tools and Applications, vol. 80, no. 4, pp. 5423-5447, 2021.

[19] S. Latha and D. Samiappan, "Despeckling of carotid artery ultrasound images with a calculus approach," Current Medical Imaging Formerly Current Medical Imaging Reviews, vol. 15, no. 4, pp. 414-426, 2019.

[20] K. Selvakuberan, D. Kayathiri, B. Harini, and M. I. Devi, “An efficient feature selection method for classification in health care systems using machine learning techniques,"vol. 4, pp. 223-226, in Proceedings of the IEEE 2011 3rd International Conference on Electronics Computer Technology, vol. 4, IEEE, Kanyakumari, India, 8 April 2011.

[21] N. Emanet, H. R. Öz, N. Bayram, and D. Delen, "A comparative analysis of machine learning methods for classification type decision problems in healthcare," Decision Analytics, vol. 1, no. 1, pp. 1-20, 2014 Dec.

[22] E. Gambhir, R. Jain, A. Gupta, and U. Tomer, "Regression analysis of COVID-19 using machine learning algorithms," in Proceedings of the IEEE 2020 International conference on smart electronics and communication (ICOSEC), pp. 65-71, IEEE, Trichy, India, 10 September 2020.

[23] P. S. Yeoh, K. W. Lai, S. L. Goh et al., "Emergence of deep learning in knee osteoarthritis diagnosis," Computational 
Intelligence and Neuroscience, vol. 2021, Article ID 4931437, 20 pages, 2021.

[24] S. Arivazhagan and S. V. Ligi, "Mango leaf diseases identification using convolutional neural network," International Journal of Pure and Applied Mathematics, vol. 120, no. 6, pp. 11067-11079, 2018.

[25] V. Yemelyanov, S. Chernyi, N. Yemelyanova, and V. Varadarajan, "Application of neural networks to forecast changes in the technical condition of critical production facilities," Computers \& Electrical Engineering, vol. 93, Article ID 107225, 2021.

[26] D. Samiappan and V. Chakrapani, "Classification of carotid artery abnormalities in ultrasound images using an artificial neural classifier," The International Arab Journal of Information Technology, vol. 13, no. 6A, pp. 756-762, 2016.

[27] M. Sandler, A. Howard, M. Zhu, A. Zhmoginov, and L. C. Chen, "Mobilenetv2: inverted residuals and linear bottlenecks," in Proceedings of the Proc IEEE Conf Comput Vis Pattern Recognit, pp. 4510-4520, IEEE, Salt Lake City, UT, USA, 18 June 2018.

[28] D. H. Hubel and T. N. Wiesel, "Receptive fields of single neurones in the cat's striate cortex," The Journal of Physiology, vol. 148, no. 3, pp. 574-591, 1959.

[29] K. Fukushima, "Neocognitron: a self-organizing neural network model for a mechanism of pattern recognition unaffected by shift in position," Biological Cybernetics, vol. 36, no. 4, pp. 193-202, 1980.

[30] Y. LeCun, "The MNIST database of handwritten digits," 1998, http://yann. lecun. com/exdb/mnist/.

[31] A. Krizhevsky, I. Sutskever, and G. E. Hinton, "Imagenet classification with deep convolutional neural networks," Advances in Neural Information Processing Systems, vol. 25, pp. 1097-1105, 2012.

[32] V. Nair and G. E. Hinton, "Rectified linear units improve restricted Boltzmann machines," InIcml, 2010.

[33] K. Simonyan and A. Zisserman, "Very deep convolutional networks for large-scale image recognition," 2014, https:// arxiv.org/abs/1409.1556.

[34] C. Szegedy, W. Liu, Y. Jia et al., "Going deeper with convolutions," in Proceedings of the Proc IEEE Conf Comput Vis Pattern Recognit, pp. 1-9, IEEE, Boston, MA, 7 June 2015.

[35] S. Liu and W. Deng, "Very deep convolutional neural network based image classification using small training sample size," in Proceedings of the 2015 3rd IAPR (ACPR), pp. 730-734, IEEE, Kuala Lumpur, Malaysia, 3 November 2015.

[36] K. He, X. Zhang, S. Ren, and J. Sun, "Deep residual learning for image recognition," in Proceedings of the Proc IEEE Conf Comput Vis Pattern Recognit, pp. 770-778, IEEE, Las Vegas, NV, USA, 27 June 2016.

[37] S. Zagoruyko and N. Komodakis, "Wide residual networks," 2016, https://arxiv.org/abs/1605.07146.

[38] K. He, X. Zhang, S. Ren, and J. Sun, "Identity mappings in deep residual networks," Computer Vision - ECCV 2016, Springer, vol. 9908, pp. 630-645, Cham, 2016.

[39] F. Chollet, "Xception: deep learning with depthwise separable convolutions," in Proceedings of the Proc IEEE Conf Comput Vis Pattern Recognit, pp. 1251-1258, IEEE, Honolulu, HI, USA, 21 June 2017.

[40] A. G. Howard, M. Zhu, B. Chen et al., "Mobilenets: efficient convolutional neural networks for mobile vision applications," 2017, https://arxiv.org/abs/1704.04861.

[41] S. Xie, R. Girshick, P. Dollár, Z. Tu, and K. He, "Aggregated residual transformations for deep neural networks," in
Proceedings of the Proc IEEE Conf Comput Vis Pattern Recognit, pp. 1492-1500, IEEE, Honolulu, HI, USA, 21 July 2017.

[42] D. M. Blei, A. Y. Ng, and M. I. Jordan, "Latent dirichlet allocation," Journal of Machine Learning Research, vol. 3, pp. 993-1022, 2003.

[43] J. Hu, L. Shen, and G. Sun, "Squeeze-and-excitation networks," in Proceedings of the Proc IEEE Conf Comput Vis Pattern Recognit, pp. 7132-7141, Salt Lake City, UT, USA, 18 June 2018.

[44] X. Zhang, S. Huang, X. Zhang, W. Wang, Q. Wang, and D. Yang, "Residual inception: a new module combining modified residual with inception to improve network performance," in Proceedings of the 2018 25th IEEE (ICIP), pp. 3039-3043, IEEE, Athens, Greece, 7 October 2018.

[45] B. Zoph, V. Vasudevan, J. Shlens, and Q. V. Le, "Learning transferable architectures for scalable image recognition," in Proceedings of the Proc IEEE Conf Comput Vis Pattern Recognit, pp. 8697-8710, IEEE, Salt Lake City, UT, USA, 18 June 2018.

[46] M. Tan and Q. Le, "Efficientnet: rethinking model scaling for convolutional neural networks," Mach Learn Based Proced, PMLR, vol. 97, pp. 6105-6114, 2019.

[47] A. Brock, S. De, S. L. Smith, and K. Simonyan, "High-performance large-scale image recognition without normalization," https://arxiv.org/abs/2102.06171 2021.

[48] R. R. Selvaraju, M. Cogswell, A. Das, R. Vedantam, D. Parikh, and D. Batra, "Grad-cam: visual explanations from deep networks via gradient-based localization," in Proceedings of the IEEE Int Conf Comput Vis, pp. 618-626, IEEE, Venice, Italy, 22 October 2017.

[49] H. Wang, R. Naidu, J. Michael, and S. S. Kundu, "SS-CAM: smoothed Score-CAM for sharper visual feature localization," 2020, https://arxiv.org/abs/2006.14255.

[50] H. Alshazly, C. Linse, E. Barth, and T. Martinetz, "Explainable COVID-19 detection using chest CT scans and deep learning," Sensors, vol. 21, no. 2, p. 455, 2021.

[51] R. K. Singh, R. Pandey, and R. N. Babu, "COVIDScreen: explainable deep learning framework for differential diagnosis of COVID-19 using chest X-Rays," Neural Computing \& Applications, vol. 8, pp. 1-22, 2021.

[52] C. Shorten, T. M. Khoshgoftaar, and B. Furht, "Deep learning applications for COVID-19," J Big Data, vol. 8, pp. 1-54, 2021.

[53] F. Shi, J. Wang, J. Shi et al., "Review of artificial intelligence techniques in imaging data acquisition, segmentation and diagnosis for COVID-19," IEEE Rev Biomed Eng, vol. 14, 2020.

[54] S. A. Harmon, T. H. Sanford, S. Xu et al., "Artificial intelligence for the detection of COVID-19 pneumonia on chest CT using multinational datasets," Nature Communications, vol. 11, pp. 4080-4087, 2020.

[55] X. Ouyang, J. Huo, L. Xia et al., "Dual-sampling attention network for diagnosis of COVID-19 from community acquired pneumonia," IEEE Transactions on Medical Imaging, vol. 39, no. 8, pp. 2595-2605, 2020.

[56] X. Wu, H. Hui, M. Niu et al., "Deep learning-based multiview fusion model for screening 2019 novel coronavirus pneumonia: a multicentre study," European Journal of Radiology, vol. 128, Article ID 109041, 2020.

[57] A. A. Ardakani, A. R. Kanafi, U. R. Acharya, N. Khadem, and A. Mohammadi, "Application of deep learning technique to manage COVID-19 in routine clinical practice using CT 
images: results of 10 convolutional neural networks," Computers in Biology and Medicine, vol. 121, p. 103795, 2020.

[58] L. Sun, Z. Mo, F. Yan et al., "Adaptive feature selection guided deep forest for COVID-19 classification with chest ct," IEEE Journal of Biomedical and Health Informatics, vol. 24, no. 10, pp. 2798-2805, 2020.

[59] A. Narin, C. Kaya, and Z. Pamuk, "Automatic detection of coronavirus disease (COVID-19) using x-ray images and deep convolutional neural networks," Pattern Analysis and Applications : PAA, vol. 24, no. 3, pp. 1-14, 2021.

[60] J. P. Cohen, P. Morrison, L. Dao, K. Roth, T. Q. Duong, and M. Ghassemi, "COVID-19 image data collection: prospective predictions are the future," 2020, https://github.com/ ieee8023/covid-chestxray-dataset.

[61] X. Wang, Y. Peng, L. Lu, Z. Lu, M. Bagheri, and R. M. Summers, "Chestx-ray8: hospital-scale chest X-ray database and benchmarks on weakly-supervised classification and localization of common thorax diseases," in Proceedings of the Proc IEEE Conf Comput Vis Pattern Recognit, pp. 2097-2106, IEEE, Honolulu, HI, USA, 21 July 2017.

[62] P. Mooney, "Chest x-ray images (pneumonia)," 2018, https:// www.kaggle.com/paultimothymooney/chest-xraypneumonia.

[63] J. Zhang, Y. Xie, Y. Li, C. Shen, and Y. Xia, "COVID-19 screening on chest $\mathrm{X}$-ray images using deep learning based anomaly detection," p. 27, 2020, https://arxiv.org/abs/2003. 12338.

[64] B. Abraham and M. S. Nair, "Computer-aided detection of COVID-19 from X-ray images using multi-CNN and Bayesnet classifier," Biocybernetics and Biomedical Engineering, vol. 40, no. 4, pp. 1436-1445, 2020.

[65] D. S. Kermany, M. Goldbaum, W. Cai et al., "Identifying medical diagnoses and treatable diseases by image-based deep learning," Cell, vol. 172, no. 5, pp. 1122-1131, 2018.

[66] A. M. Dadario, "COVID-19 X rays," 2020, https://www. kaggle. com/andrewmvd/convid19-X-rays.

[67] P. Autee, S. Bagwe, V. Shah, and K. Srivastava, "StackNetDenVIS: a multi-layer perceptron stacked ensembling approach for COVID-19 detection using X-ray images," Physical and Engineering Sciences in Medicine, vol. 43, no. 4, pp. 1399-1414, 2020.

[68] L. Wang, Z. Q. Lin, and A. Wong, "Covid-net: a tailored deep convolutional neural network design for detection of COVID-19 cases from chest x-ray images," Scientific Reports, vol. 10, pp. 1-12, 2020.

[69] M. E. H. Chowdhury, T. Rahman, A. Khandakar et al., "Can AI help in screening viral and COVID-19 pneumonia?" IEEE Access, vol. 8, pp. 132665-132676, 2020.

[70] L. Li, L. Qin, Z. Xu et al., "Artificial intelligence distinguishes COVID-19 from community acquired pneumonia on chest CT," Radiology, 2020.

[71] J. Wang, Y. Bao, Y. Wen et al., "Prior-attention residual learning for more discriminative COVID-19 screening in CT images," IEEE Transactions on Medical Imaging, vol. 39, no. 8, pp. 2572-2583, 2020.

[72] A. M. Hasan, M. M. Al-Jawad, H. A. Jalab, H. Shaiba, R. W. Ibrahim, and A. a. R. AL-Shamasneh, "Classification of COVID-19 coronavirus, pneumonia and healthy lungs in ct scans using q-deformed entropy and deep learning features," Entropy, vol. 22, no. 5, p. 517, 2020.

[73] C. Butt, J. Gill, D. Chun, and B. A. Babu, "Deep learning system to screen coronavirus disease 2019 pneumonia," Applied Intelligence, vol. 6, pp. 1-7, 2020.
[74] Y. Song, S. Zheng, L. Li et al., "Deep learning enables accurate diagnosis of novel coronavirus (COVID-19) with CT images," IEEE/ACM Transactions on Computational Biology and Bioinformatics, vol. 18, no. 6, 2021.

[75] M. Toğaçar, B. Ergen, and Z. Cömert, "COVID-19 detection using deep learning models to exploit Social Mimic Optimization and structured chest X-ray images using fuzzy color and stacking approaches," Computers in Biology and Medicine, vol. 121, Article ID 103805, 2020.

[76] A. Chung, "Figure 1 COVID-19 chest x-ray data initiative," 2020, https://github.com/agchung/Figure1-COVIDchestxray-dataset.

[77] A. Chung, "Actualmed COVID-19 chest x-ray data initiative," 2020, https://github.com/agchung/ActualmedCOVID-chestxray-dataset.

[78] RS, “COVID-19 radiography database," 2020, https://www. kaggle.com/tawsifurrahman/covid19-radiography-\%20database.

[79] RS, "RSNA pneumonia detection challenge," https://www. kaggle.com/c/rsna-pneumonia-detection-challenge/data.

[80] M. Nishio, S. Noguchi, H. Matsuo, and T. Murakami, "Automatic classification between COVID-19 pneumonia, non-COVID-19 pneumonia, and the healthy on chest X-ray image: combination of data augmentation methods," Scientific Reports, vol. 10, pp. 17532-17536, 2020.

[81] M. Canayaz, "MH-COVIDNet: diagnosis of COVID-19 using deep neural networks and meta-heuristic-based feature selection on X-ray images," Biomedical Signal Processing and Control, vol. 64, Article ID 102257, 2021.

[82] Y. E. Almalki, A. Qayyum, M. Irfan et al., "A novel method for COVID-19 diagnosis using artificial intelligence in chest X-ray images," Healthcare, vol. 9, no. 5, p. 522, 2021.

[83] [dataset], "Radiopaedia," 2020, https://radiopaedia.org/ search?lang $=$ uspage $=4 \mathrm{q}=$ covid +19 scope $=$ allutf $8=\% e 2 \% 9 c \%$ 93.

[84] R. Hu, G. Ruan, S. Xiang, M. Huang, Q. Liang, and J. Li, Automated Diagnosis of COVID-19 Using Deep Learning and Data Augmentation on Chest Ct, medRxiv, 2020.

[85] [dataset], "COVID-19 database," 2020, https://www.sirm. org/category/senzacategoria/COVID-19/.

[86] L. Sarker, M. M. Islam, T. Hannan, and Z. Ahmed, "Coviddensenet: a deep learning architecture to detect COVID-19 from chest radiology images," Preprints, 2020.

[87] T. Ozturk, M. Talo, E. A. Yildirim, U. B. Baloglu, O. Yildirim, and U. Rajendra Acharya, "Automated detection of COVID19 cases using deep neural networks with X-ray images," Computers in Biology and Medicine, vol. 121, Article ID 103792, 2020.

[88] T. Mahmud, M. A. Rahman, and S. A. Fattah, "CovXNet: a multi-dilation convolutional neural network for automatic COVID-19 and other pneumonia detection from chest X-ray images with transferable multi-receptive feature optimization," Computers in Biology and Medicine, vol. 122, Article ID 103869, 2020.

[89] M. Z. Alom, M. M. Rahman, M. S. Nasrin, T. M. Taha, and V. K. Asari, "Covid_mtnet: COVID-19 detection with multitask deep learning approaches," 2020, https://arxiv.org/abs/ 2004.03747

[90] D. N. Vinod and S. R. S. Prabaharan, "Data science and the role of Artificial Intelligence in achieving the fast diagnosis of COVID-19," Chaos, Solitons \& Fractals, vol. 140, p. 110182, 2020.

[91] V. Perumal, V. Narayanan, and S. J. S. Rajasekar, "Detection of COVID-19 using CXR and CT images using Transfer 
Learning and Haralick features," Applied Intelligence, vol. 51, no. 1, pp. 341-358, 2021.

[92] D. Singh, V. Kumar, M. Vaishali, and M. Kaur, "Classification of COVID-19 patients from chest CT images using multi-objective differential evolution-based convolutional neural networks," European Journal of Clinical Microbiology \& Infectious Diseases, vol. 39, no. 7, pp. 1379-1389, 2020.

[93] Z. Xue, D. You, S. Candemir et al., "Chest X-ray image view classification," in Proceedings of the 2015 IEEE 28th International Symposium on Computer-Based Medical Systems, pp. 66-71, IEEE, Sao Carlos, Brazil, 22 June 2015.

[94] M. Irfan, M. A. Iftikhar, S. Yasin et al., "Role of hybrid deep neural networks (HDNNs), computed tomography, and chest X-rays for the detection of COVID-19," International Journal of Environmental Research and Public Health, vol. 18, no. 6, p. 3056, 2021.

[95] J. Chen, L. Wu, J. Zhang et al., "Deep learning-based model for detecting 2019 novel coronavirus pneumonia on highresolution computed tomography," Scientific Reports, vol. 10, no. 1, pp. 1-11, 2020.

[96] D. Demner-Fushman, M. D. Kohli, M. B. Rosenman et al., "Preparing a collection of radiology examinations for distribution and retrieval," Journal of the American Medical Informatics Association, vol. 23, no. 2, pp. 304-310, 2016.

[97] T. D. Pham, "A comprehensive study on classification of COVID-19 on computed tomography with pretrained convolutional neural networks," Scientific Reports, vol. 10, pp. 16942-16948, 2020.

[98] H. Panwar, P. K. Gupta, M. K. Siddiqui, R. MoralesMenendez, and V. Singh, "Application of deep learning for fast detection of COVID-19 in X-Rays using nCOVnet," Chaos, Solitons \& Fractals, vol. 138, Article ID 109944, 2020.

[99] M. Rahimzadeh, A. Attar, and S. M. Sakhaei, "A fully automated deep learning-based network for detecting COVID19 from a new and large lung ct scan dataset," Biomedical Signal Processing and Control, vol. 68, Article ID 102588, 2021.

[100] S. Albahli, "Efficient GAN-based Chest Radiographs (CXR) augmentation to diagnose coronavirus disease pneumonia," International Journal of Medical Sciences, vol. 17, no. 10, pp. 1439-1448, 2020.

[101] Y. Cao, Z. Xu, J. Feng et al., "Longitudinal assessment of COVID-19 using a deep learning-based quantitative CT pipeline: illustration of two cases," Radiology: Cardiothoracic Imaging, vol. 2, no. 2, Article ID e200082, 2020.

[102] B. Wang, S. Jin, Q. Yan et al., "AI-assisted CT imaging analysis for COVID-19 screening: building and deploying a medical AI system," Applied Soft Computing, vol. 98, Article ID 106897, 2021.

[103] L. Huang, R. Han, T. Ai et al., "Serial quantitative chest CT assessment of COVID-19: a deep learning approach," Radiology: Cardiothoracic Imaging, vol. 2, no. 2, Article ID e200075, 2020.

[104] F. Shan, Y. Gao, J. Wang et al., "Lung infection quantification of COVID-19 in CT images with deep learning," 2020, https://arxiv.org/abs/2003.04655.

[105] N. Sverzellati, C. J. Ryerson, G. Milanese et al., "Chest x-ray or CT for COVID-19 pneumonia? Comparative study in a simulated triage setting," European Respiratory Journal, vol. 58, 2021.

[106] S. Wang, Y. Zha, W. Li et al., "A fully automatic deep learning system for COVID-19 diagnostic and prognostic analysis," European Respiratory Journal, vol. 56, 2020.
[107] Z. Feng, Q. Yu, S. Yao et al., "Early prediction of disease progression in COVID-19 pneumonia patients with chest CT and clinical characteristics," Nature Communications, vol. 11, pp. 4968-4969, 2020.

[108] W. Liang, H. Liang, L. Ou et al., "Development and validation of a clinical risk score to predict the occurrence of critical illness in hospitalized patients with COVID-19," JAMA Internal Medicine, vol. 180, no. 8, pp. 1081-1089, 2020.

[109] Q. Wu, S. Wang, L. Li et al., "Radiomics Analysis of Computed Tomography helps predict poor prognostic outcome in COVID-19," Theranostics, vol. 10, no. 16, pp. 7231-7244, 2020.

[110] J. P. Cohen, L. Dao, K. Roth et al., "Predicting COVID-19 pneumonia severity on chest $\mathrm{x}$-ray with deep learning," Cureus, vol. 12, p. e9448, 2020.

[111] G. Shih, C. C. Wu, S. S. Halabi et al., "Augmenting the national institutes of health chest radiograph dataset with expert annotations of possible pneumonia," Radiology: Artificial Intelligence, vol. 1, no. 1, Article ID e180041, 2019.

[112] J. Irvin, P. Rajpurkar, M. Ko et al., "Chexpert: a large chest radiograph dataset with uncertainty labels and expert comparison," Proceedings of the AAAI Conference on Artificial Intelligence, vol. 33, pp. 590-597, 2019.

[113] A. Majkowska, S. Mittal, D. F. Steiner et al., "Chest radiograph interpretation with deep learning models: assessment with radiologist-adjudicated reference standards and population-adjusted evaluation," Radiology, vol. 294, no. 2, pp. 421-431, 2020.

[114] A. E. Johnson, T. J. Pollard, N. R. Greenbaum et al., "MIMICCXR-JPG, a large publicly available database of labeled chest radiographs," 2019, https://arxiv.org/abs/1901.07042.

[115] A. Bustos, A. Pertusa, J.-M. Salinas, and M. de la IglesiaVayá, "Padchest: a large chest $\mathrm{x}$-ray image dataset with multilabel annotated reports," Medical Image Analysis, vol. 66, Article ID 101797, 2020.

[116] J. Zhu, B. Shen, A. Abbasi, M. Hoshmand-Kochi, H. Li, and T. Q. Duong, "Deep transfer learning artificial intelligence accurately stages COVID-19 lung disease severity on portable chest radiographs," PLoS One, vol. 15, no. 7, Article ID e0236621, 2020.

[117] S. Tabik, A. Gomez-Rios, J. L. Martin-Rodriguez et al., "COVIDGR dataset and COVID-SDNet methodology for predicting COVID-19 based on Chest X-Ray images," IEEE Journal of Biomedical and Health Informatics, vol. 24, no. 12, pp. 3595-3605, 2020.

[118] M. Shorfuzzaman and M. S. Hossain, "MetaCOVID: a Siamese neural network framework with contrastive loss for n-shot diagnosis of COVID-19 patients," Pattern Recognition, vol. 113, Article ID 107700, 2021.

[119] I. Ozsahin, B. Sekeroglu, M. S. Musa, M. T. Mustapha, and D. Uzun Ozsahin, "Review on diagnosis of COVID-19 from chest CT images using artificial intelligence," Comput Math Methods Med, vol. 2020, Article ID 9756518, 10 pages, 2020.

[120] A. Shoeibi, M. Khodatars, R. Alizadehsani et al., "Automated detection and forecasting of COVID-19 using deep learning techniques: a review," 2020, https://arxiv.org/abs/2007. 10785.

[121] D. Chicco and G. Jurman, “The advantages of the Matthews correlation coefficient (MCC) over F1 score and accuracy in binary classification evaluation," BMC Genomics, vol. 21, pp. 6-3, 2020

[122] M. H. Alsharif, Y. H. Alsharif, K. Yahya, O. A. Alomari, M. A. Albreem, and A. Jahid, "Deep learning applications to combat the dissemination of COVID-19 disease: a review," 
European Review for Medical and Pharmacological Sciences, vol. 24, pp. 11455-11460, 2020.

[123] S. K. Joy, F. Ahmed, M. Humaira et al., "A comprehensive survey of COVID-19 detection using medical images," SN Comput Sci.vol. 2, 2020.

[124] H. Alghamdi, G. Amoudi, S. Elhag, K. Saeedi, and J. Nasser, "Deep learning approaches for detecting COVID-19 from chest X-ray images: a survey,” IEEE Access, vol. 9, 2021.

[125] M. M. Islam, F. Karray, R. Alhajj, and J. Zeng, "A review on deep learning techniques for the diagnosis of novel coronavirus (COVID-19)," IEEE Access, vol. 9, pp. 30551-30572, 2021.

[126] A. Sriram, M. Muckley, K. Sinha et al., "COVID-19 deterioration prediction via self-supervised representation learning and multi-image prediction," 2021, https://arxiv. org/abs/2101.04909.

[127] A. Artetxe, A. Beristain, and M. Graña, "Predictive models for hospital readmission risk: a systematic review of methods," Computer Methods and Programs in Biomedicine, vol. 164, pp. 49-64, 2018.

[128] X. Min, B. Yu, and F. Wang, "Predictive modeling of the hospital readmission risk from patients' claims data using machine learning: a case study on COPD," Scientific Reports, vol. 9, no. 1, pp. 1-0, 2019.

[129] K. Teo, C. W. Yong, F. Muhamad et al., "The promise for reducing healthcare cost with predictive model: an analysis with quantized evaluation metric on readmission," J Healthcare Eng, vol. 2021, Article ID 9208138, 10 pages, 2021.

[130] J. Donzé, D. Aujesky, D. Williams, and J. L. Schnipper, "Potentially avoidable 30-day hospital readmissions in medical patients: derivation and validation of a prediction model," JAMA Internal Medicine, vol. 173, no. 8, pp. 632-638, 2013.

[131] O. A. UyaroĞlu, N. Ç BaŞaran, L. ÖziŞik et al., “Thirty-day readmission rate of COVID-19 patients discharged from a tertiary care university hospital in Turkey: an observational, single-center study," International Journal for Quality in Health Care : Journal of the International Society for Quality in Health Care, vol. 33, no. 1, p. 144, 2021.

[132] S. Sotoodeh Ghorbani, N. Taherpour, S. Bayat, H. Ghajari, P. Mohseni, and S. S. Hashemi Nazari, "Epidemiologic characteristics of cases with reinfection, recurrence, and hospital readmission due to COVID-19: a systematic review and meta-analysis," Journal of Medical Virology, vol. 94, no. 1, pp. 44-53, 2021.

[133] P. Goyal, M. Caron, B. Lefaudeux et al., "Self-supervised pretraining of visual features in the wild," 2021, https://arxiv. org/abs/2103.01988.

[134] H. Pham, Z. Dai, Q. Xie, M. T. Luong, and Q. V. Le, "Meta pseudo labels,” 2020, https://arxiv.org/abs/2003.10580.

[135] Y. Oh, H. Jung, J. Park, and M. S. Kim, "EVET: enhancing visual explanations of deep neural networks using image transformations," in Proceedings of the IEEE Winter Conf Appl Comput Vis, pp. 3579-3587, IEEE, Waikoloa, HI, USA, 3 January 2021.

[136] K. Vinogradova, A. Dibrov, and G. Myers, "Towards interpretable semantic segmentation via gradient-weighted class activation mapping," 2020, https://arxiv.org/abs/2002. 11434.

[137] B. Zhou, A. Khosla, A. Lapedriza, A. Oliva, and A. Torralba, "Learning deep features for discriminative localization," Proc IEEE Conf Comput Vis Pattern Recognit, pp. 2921-2929, 2016.
[138] A. A. Taha and A. Hanbury, "Metrics for evaluating 3D medical image segmentation: analysis, selection, and tool," BMC Medical Imaging, vol. 15, pp. 29-28, 2015.

[139] R. Roelofs, Measuring Generalization and Overfitting in Machine Learning, Doctoral dissertation, UC Berkeley), 2019.

[140] X. He, S. Wang, G. Ying, J. Zhang, and X. Chu, "Efficient multi-objective evolutionary 3D neural architecture search for COVID-19 detection with chest CT scans.," 2021, https:// arxiv.org/abs/2101.10667.

[141] Z. Li, W. Zhao, F. Shi et al., "A novel multiple instance learning framework for COVID-19 severity assessment via data augmentation and self-supervised learning," Medical Image Analysis, vol. 69, Article ID 101978, 2021.

[142] I. Singh, H. Zhou, K. Yang, M. Ding, B. Lin, and P. Xie, "Differentially-private federated neural architecture search," 2020, https://arxiv.org/abs/2006.10559.

[143] M. Xu, Y. Zhao, K. Bian, G. Huang, Q. Mei, and X. Liu, "Eural architecture search over decentralized data," in $N$, https://arxiv.org/abs/2002.06352, 2020.

[144] A. Priyanshu and R. Naidu, "FedPandemic: a cross-device federated learning approach towards elementary prognosis of diseases during a pandemic," 2021, https://arxiv.org/abs/ 2104.01864.

[145] G. Yadav, S. Maheshwari, and A. Agarwal, "Contrast limited adaptive histogram equalization based enhancement for real time video system," in Proceedings of the 2014 International Conference on Advances in Computing, Communications and Informatics, pp. 2392-2397, IEEE, Delhi, India, 24 September 2014.

[146] S. M. Pizer, E. P. Amburn, J. D. Austin et al., "Adaptive histogram equalization and its variations," Computer Vision, Graphics, and Image Processing, vol. 39, no. 3, pp. 355-368, 1987.

[147] T. L. Veldhuizen and M. E. Jernigan, "Grid filters for local nonlinear image restoration,"vol. 5, pp. 2885-2888, in Proceedings of the 1998 IEEE International Conference on Acoustics, Speech and Signal Processing, (Cat. No. 98CH36181), vol. 5, pp. 2885-2888, IEEE, Seattle, WA, USA, 15 May 1998.

[148] T. M. Lehmann, C. Gonner, and K. Spitzer, "Survey: interpolation methods in medical image processing," IEEE Transactions on Medical Imaging, vol. 18, no. 11, pp. 10491075, 1999.

[149] J. Tian, Y. Wang, X. Dai, and X. Zhang, "Medical image processing and analysis," in Mol Imaging, pp. 415-469, Springer, Berlin, Heidelberg, 2013.

[150] D. S. Prabha and J. S. Kumar, "Performance analysis of image smoothing methods for low level of distortion," in Proceedings of the 2016 IEEE International Conference on Advances in Computer Applications, pp. 372-376, IEEE, Coimbatore, India, 24 October 2016.

[151] M. Kociołek, M. Strzelecki, and R. Obuchowicz, "Does image normalization and intensity resolution impact texture classification?" Computerized Medical Imaging and Graphics, vol. 81, Article ID 101716, 2020.

[152] M. A. Gungor, "A comparative study on wavelet denoising for high noisy CT images of COVID-19 disease," Optik, vol. 235, Article ID 166652, 2021.

[153] A. Waheed, M. Goyal, D. Gupta, A. Khanna, F. Al-Turjman, and P. R. Pinheiro, "Covidgan: data augmentation using auxiliary classifier gan for improved COVID-19 detection," IEEE Access, vol. 8, pp. 91916-91923, 2020. 
[154] D. Samiappan, S. Latha, T. R. Rao, D. Verma, and C. Sriharsha, "Enhancing machine learning aptitude using significant cluster identification for augmented image refining," International Journal of Pattern Recognition and Artificial Intelligence, vol. 34, no. 09, Article ID 2051009, 2020.

[155] A. Mobiny, P. A. Cicalese, S. Zare et al., "Radiologist-level COVID-19 detection using ct scans with detail-oriented capsule networks," 2004, https://arxiv.org/abs/2004.07407.

[156] A. Oulefki, S. Agaian, T. Trongtirakul, and A. Kassah Laouar, "Automatic COVID-19 lung infected region segmentation and measurement using CT-scans images," Pattern Recognition, vol. 114, Article ID 107747, 2021.

[157] S. Park, G. Kim, Y. Oh et al., "Vision transformer for COVID-19 CXR diagnosis using chest X-ray feature corpus," 2021, https://arxiv.org/abs/2103.07055.

[158] G. Maguolo and L. Nanni, "A critic evaluation of methods for COVID-19 automatic detection from x-ray images," Information Fusion, vol. 76, 2021.

[159] P. Rajpurkar, J. Irvin, K. Zhu et al., "Chexnet: radiologistlevel pneumonia detection on chest $\mathrm{x}$-rays with deep learning," 2017, https://arxiv.org/abs/1711.05225.

[160] A. S. Al-Waisy, S. Al-Fahdawi, M. A. Mohammed et al., "COVID-CheXNet: hybrid deep learning framework for identifying COVID-19 virus in chest X-rays images," Soft Comput, pp. 1-6, 2020. 